\title{
Biodegradable polyelectrolyte/magnetite capsules for MR imaging and magnetic targeting of tumors
}

\author{
Yulia Svenskaya ${ }^{{ }^{\boxplus *}}$, Francesca Garello ${ }^{2 *}$, Ekaterina Lengert' ${ }^{1}$, Anastasiia Kozlova ${ }^{3}$, Roman Verkhovskii ${ }^{3}$, \\ Valeria Bitonto², Maria Rosaria Ruggiero², Sergey German ${ }^{4,5}$, Dmitry Gorin ${ }^{5}$, Enzo Terreno ${ }^{2}$
}

1. Remote Controlled Systems for Theranostics laboratory, Research and Educational Institute of Nanostructures and Biosystems, Saratov State University, 410012 Saratov, Russia.

2. Molecular and Preclinical Imaging Centres, Department of Molecular Biotechnology and Health Sciences, University of Torino, 10126 Torino, Italy.

3. Biomedical Photoacoustics Laboratory, Saratov State University, 410012 Saratov, Russia.

4. Laboratory of Optics and Spectroscopy of Nanoobjects, Institute of Spectroscopy of the RAS, Troitsk 108840, Russia.

5. Center of Photonics and Quantum Materials, Skolkovo Institute of Science and Technology, 143026 Moscow, Russia.

* Yu. Svenskaya and F. Garello contributed equally to this work.

$\triangle$ Corresponding authors: Yulia Svenskaya, E-mail: svenskaya@info.sgu.ru; Enzo Terreno, E-mail: enzo.terreno@unito.it

(C) The author(s). This is an open access article distributed under the terms of the Creative Commons Attribution License (https://creativecommons.org/licenses/by/4.0/). See http://ivyspring.com/terms for full terms and conditions.

Received: 2021.02.18; Accepted: 2021.03.15; Published: 2021.04.02

\begin{abstract}
Rationale: The tireless research for effective drug delivery approaches is prompted by poor target tissue penetration and limited selectivity against diseased cells. To overcome these issues, various nanoand micro-carriers have been developed so far, but some of them are characterized by slow degradation time, thus hampering repeated drug administrations. The aim of this study was to pursue a selective delivery of magnetic biodegradable polyelectrolyte capsules in a mouse breast cancer model, using an external magnetic field.

Methods: Four different kinds of magnetic polyelectrolyte capsules were fabricated via layer-by-layer assembly of biodegradable polymers on calcium carbonate templates. Magnetite nanoparticles were embedded either into the capsules' shell (sample S) or both into the shell and the inner volume of the capsules (samples $\mathrm{C}_{\mathrm{n}} \mathrm{S}$, where $\mathrm{n}$ is the number of nanoparticle loading cycles). Samples were first characterized in terms of their relaxometric and photosedimentometric properties. In vitro magnetic resonance imaging (MRI) experiments, carried out on RAW 264.7 cells, allowed the selection of two lead samples that proceeded for the in vivo testing on a mouse breast cancer model. In the set of in vivo experiments, an external magnet was applied for 1 hour following the intravenous injection of the capsules to improve their delivery to tumor, and MRI scans were acquired at different time points post administration.

Results: All samples were considered non-cytotoxic as they provided more than $76 \%$ viability of RAW 264.7 cells upon $2 \mathrm{~h}$ incubation. Sample $\mathrm{S}$ appeared to be the most efficient in terms of $\mathrm{T}_{2}-\mathrm{MRI}$ contrast, but the less sensitive to external magnet navigation, since no difference in MRI signal with and without the magnet was observed. On the other side, sample $\mathrm{C}_{6} \mathrm{~S}$ was efficiently delivered to the tumor tissue, with a three-fold $T_{2}-M R I$ contrast enhancement upon the external magnet application. The effective magnetic targeting of $\mathrm{C}_{6} \mathrm{~S}$ capsules was also confirmed by the reduction in $\mathrm{T}_{2}-\mathrm{MRI}$ contrast in spleen if compared with the untreated with magnet mice values, and the presence of dense and clustered iron aggregates in tumor histology sections even $48 \mathrm{~h}$ after the magnetic targeting.

Conclusion: The highlighted strategy of magnetic biodegradable polyelectrolyte capsules' design allows for the development of an efficient drug delivery system, which through an MRI-guided externally controlled navigation may lead to a significant improvement of the anticancer chemotherapy performance.
\end{abstract}

Key words: magnetically-guided drug delivery systems; polyelectrolyte submicron capsules; magnetite nanoparticles; magnetic resonance imaging; targeted drug delivery. 


\section{Introduction}

The search for effective drug delivery approaches is driven by the observation that many therapeutic agents failed due to their limited ability to reach the target tissue and their poor selectivity against diseased cells. Additionally, also the drug availability at the pathological site, determined by the release of the drug from its carrier, is a critical step. A wide variety of carriers have been investigated so far, including lipid-based delivery systems [1] and conjugates [2], polymeric [3, 4] and inorganic particles $[5,6]$, "host-guest" supramolecular adducts $[7,8]$, and naturally-occurring systems like lipoproteins [9, 10], proteins [11, 12], peptides [13, 14], viral capsids $[15,16]$, bacteria $[17,18]$, cells $[19,20]$ and extracellular vesicles/exosomes [21]. Drugs can be released from the carrier spontaneously or through specific chemical (e.g. decreased $\mathrm{pH}$, redox condition, enzymes) or physical triggering stimuli (e.g. ultrasound, heat, light, electric or magnetic fields) exploited either alone or in tandem [22-27]. However, since the amount of drug that can be encapsulated is limited and only a small percent of carriers reaches the target tissue, the efficiency of drug delivery can be controversial. Specifically, the analysis of the drug delivery studies published in 2005-2015, has shown that only $0.7 \%$ (median) of the administered nanoparticle dose was delivered to a solid tumor [28]. Such a poor efficiency is associated with the physiological barriers that a drug-carrying platform faces after its intravenous injection (for example, diffusion, flow and shear forces, aggregation, protein corona adsorption, phagocytic sequestration and renal clearance) $[29,30]$. In terms of cancer treatment, the additional limitations are attributed to the heterogeneity of tumor vasculature exhibiting the zones of both increased and sparse vascular density, hierarchical disorganization, serpentine structure and irregular branching [28]. In addition to the limited therapeutic efficiency, such off-target tissue delivery raises toxicity concerns.

Various targeting strategies enabling a site-specific drug addressing have been developed to overcome the physiological barriers. Molecular systems of all-length scales, from small molecules to cells [31, 32], and external physical stimulations, like electric and magnetic fields, ultrasound, mechanical forces, light and temperature gradients, [33, 34] allow the control of the carrier navigation.

Magnetic targeting has been widely used owing to the ability of magnetic fields to penetrate most materials [34, 35]. Moreover, magnetic fields can pass through the body safely, opening up the perspective of magnetic carrier delivery to deep tissues [36]. Both static and varying field magnet systems have been extensively studied. However, clinical trials mainly utilize permanent magnets for magnetic targeting [36]. A plenty of magnetic carrier types have been proposed and effectively applied for remotely controlled targeting purposes [37-40]. Among them, magnetic polyelectrolyte multilayer capsules represent a unique delivery system allowing for remote navigation with magnetic field and in-situ release of encapsulated material, including triggered drug release in response to physical stimuli, such as light or ultrasound [41]. Recently, the enhanced delivery effectiveness under external magnet application was demonstrated in vitro and in vivo for micron- [42, 43] and submicron-sized [44] polyelectrolyte multilayer constructs decorated with magnetite nanoparticles.

The monitoring of carrier delivery to a specific organ (or tumor in the case of anticancer therapy) is a separate challenging task. The doping of carriers by magnetite nanoparticles opens opportunities for their visualization and control of drug delivery/release steps by magnetic resonance imaging (MRI) in vivo $[44,45]$. Moreover, the degradation of a carrier can be detected through the change in MRI contrast of adjacent tissues caused by the enhancement of distance between nanoparticles upon their liberation [46]. This also provides an indirect detection of encapsulated substance release. Furthermore, initial contrast of polyelectrolyte capsules can be controlled by the change in their structure or composition [47]. By this means, an appropriate structure of such capsules can provide the ideal balance between MRI contrast and magnetic navigation property while keeping the enhanced payload ability.

Furthermore, the use of drug delivery carriers doped with magnetic nanoparticles opens up the perspectives for local magnetic hyperthermia of adjacent cancer tissues. Since alternating magnetic fields enable the heating of magnetic nanoparticles, hyperthermia in addition to drug therapeutic effect can be granted if particles are localized within the tumor [48-50]. Generation of local hyperthermia can be exploited also to induce the drug release from the delivered carriers [51-53] and to permeabilize the cell membranes, with the beneficial consequence of improving the drug diffusion in the lesion [54].

By this means, fabrication of magnetic drug-carrying containers that can provide the enhanced drug delivery efficiency together with the ability to monitor and prove this delivery is an important task in theranostics field, especially in anticancer theranostics. The aim of this study was to explore the magnetic biodegradable polyelectrolyte capsules, combining the externally controlled 
navigation and MRI visualization properties, in vivo in a breast cancer mouse model.

\section{Experimental Section}

\section{Materials}

Calcium chloride $\left(\mathrm{CaCl}_{2}\right)$, sodium carbonate $\left(\mathrm{Na}_{2} \mathrm{CO}_{3}\right)$, ethylene glycol (EG), poly-L-arginine (PA), dextran sulfate sodium salt (DS), sodium chloride $(\mathrm{NaCl})$, ethylenediaminetetraacetic acid (EDTA), iron (III) chloride hexahydrate $\left(\mathrm{FeCl}_{3}\right)$, iron (II) chloride tetrahydrate $\left(\mathrm{FeCl}_{2}\right)$, potassium ferricyanide, hydrochloric acid $(\mathrm{HCl}) 37 \%, 10 \%$ neutral buffered formalin (NBF) and nuclear fast red (NFR) were purchased from Sigma-Aldrich (USA). Nitric acid $\left(\mathrm{HNO}_{3}\right) 70 \%$ for trace metal analysis was purchased from Thermo Scientific ${ }^{\mathrm{TM}}$ (USA). Roswell Park Memorial Institute Medium (RPMI-1640), Dulbecco's Modified Eagle's Medium (DMEM), heat-inactivated fetal bovine serum (FBS), L-glutamine, trypsin/EDTA and penicillin-streptomycin mixture were purchased from Lonza (Belgium). Dulbecco's phosphate buffered saline (PBS), acridine orange (AO) and propidium iodide (PI) were purchased from Gibco (USA). Bradford Protein Assay was purchased from Bio-Rad Laboratories (USA). Milli-Q water was used in all experiments (Milli-Q Purification System, Millipore, Merck, USA).

\section{Magnetite synthesis}

The synthesis of magnetite nanoparticles (MNPs) was carried out by chemical precipitation from diand trivalent iron salts in the presence of a base. Initially, $1.3 \mathrm{~g}$ of $\mathrm{FeCl}_{3}$ and $0.48 \mathrm{~g}$ of $\mathrm{FeCl}_{2}$ were mixed and dissolved in $25 \mathrm{~mL}$ of water under room temperature, and $0.8 \mathrm{~g}$ of citric acid were dissolved in water of the same volume. Then, $170 \mathrm{~mL}$ of $0.1 \mathrm{M}$ $\mathrm{NaOH}$ were placed into the reaction cell. To remove excessive oxygen, the nitrogen was bubbled through the reaction cell, as well as the solutions of iron salts and citric acid. The iron salts were injected into the reaction cell after its heating until $40{ }^{\circ} \mathrm{C}$ with active mixing. After this, the obtained suspension was left under active mixing and nitrogen pressure for $40 \mathrm{~s}$ resulting in black sediment formation of magnetite nanoparticles. $25 \mathrm{~mL}$ of citric acid were further added to the reaction cell under constant mixing and nitrogen pressure. Dialysis of magnetic hydrosol was carried out during 3 days in a $3 \mathrm{~L}$ vial under slow mixing (50 rpm).

The size and morphology of the obtained MNPs were characterized by means of transmission electron microscopy (TEM) using a FEGTEM microscope (JEOL, Akishima, Tokyo, Japan) operating at $200 \mathrm{kV}$. For this purpose, a drop of the nanoparticle suspension was deposited onto a lacey-carbon copper grid. Image analysis and statistics were performed using Image J free software.

The hydrodynamic radius of nanoparticles was measured by dynamic light scattering (DLS) using a Zetasizer Nano ZS instrument (Malvern Instruments Ltd, Malvern, UK).

\section{Preparation of the carriers}

Magnetic polyelectrolyte capsules were fabricated via Layer-by-Layer (LbL) assembly of biodegradable polymers on calcium carbonate $\left(\mathrm{CaCO}_{3}\right)$ templates [55]. For this purpose, $\mathrm{CaCO}_{3}$ submicron particles were synthesized by precipitation from the mixture of $\mathrm{CaCl}_{2}$ and $\mathrm{Na}_{2} \mathrm{CO}_{3}$ water solutions at the EG presence [56]. Equal volumes of $0.33 \mathrm{M}$ salts were added each to EG in 2:10 volume ratio and rapidly stirred at $700 \mathrm{rpm}$ for 3 hours. The synthesized $\mathrm{CaCO}_{3}$ particles were thoroughly washed twice with water and once with ethanol, afterwards they were dried for $30 \mathrm{~min}$ at $60^{\circ} \mathrm{C}$.

Two different types of MNP-doped carriers were formed then: 1) containing MNPs in the polyelectrolyte shell - sample $S ; 2$ ) containing MNPs in both, the shell and the inner volume of the capsules samples $C_{n} S$. Different $C_{n} S$ samples were obtained depending on the amount of incorporated magnetite samples $C_{1} S, C_{2} S, C_{6} S$.

To incorporate MNPs into the inner volume of the capsules, magnetite nanoparticles were loaded into pores of $\mathrm{CaCO}_{3}$ cores before the shell deposition via freezing-induced method [57]. For this purpose, the weighted portion $(40 \mathrm{mg})$ of dried $\mathrm{CaCO}_{3}$ particles was resuspended in $2 \mathrm{~mL}$ of $1.7 \mathrm{mg} \mathrm{mL}^{-1}$ MNPs water suspension and then kept in a freezing chamber at $-20^{\circ} \mathrm{C}$ for $2 \mathrm{~h}$ under slow constant mixing with a rotator TetraQuant R-1 (TetraQuant, Russia). After that, the samples were thawed at room temperature and centrifuged at $3800 \mathrm{~g}$ for $1 \mathrm{~min}$ to separate the particles pellet. Freezing/thawing cycles were repeated 1, 2 and 6 times (samples $C_{1} S, C_{2} S, C_{6} S$, respectively).

In order to incorporate MNPs into the capsules' shell, magnetite nanoparticles were adsorbed from 0.3 $\mathrm{mg} \mathrm{mL} \mathrm{m}^{-1}$ water suspension as a shell layer. The shells were formed by self-assembly method using biocompatible PA and DS polyelectrolytes dissolved in $0.15 \mathrm{M} \mathrm{NaCl}$ at $1 \mathrm{mg} \mathrm{mL}^{-1}$ concentration. For each shell layer formation, $\mathrm{CaCO}_{3}$ particles were resuspended in $1 \mathrm{~mL}$ of the adsorbing solution, and the deposition was carried out by continuous shaking for 10 minutes. At the end of each adsorption cycle, the suspension was centrifuged for 1 minute at $3800 \mathrm{~g}$ to sediment particles and to remove a supernatant liquid phase, and then particles were triply washed with water. After consequent deposition of six layers 
(PA/DS/PA/MNPs/PA/DS), $\mathrm{CaCO}_{3}$ templates were dissolved in 0.2 M EDTA, and the suspension of capsules was triply washed with water, centrifuged at $3800 \mathrm{~g}$ for 3 minutes, and resuspended in water. Thus, different hollow MNP-doped biocompatible polyelectrolyte submicron capsules were formed (samples $\mathrm{S}, \mathrm{C}_{1} \mathrm{~S}, \mathrm{C}_{2} \mathrm{~S}$ and $\mathrm{C}_{6} \mathrm{~S}$ ).

Pure six-layered polyelectrolyte submicron capsules made of PA and DS were prepared as a control. No incorporation of MNPs was made for this sample (neither into the shell nor into the inner volume of the capsules).

The morphology of the prepared polyelectrolyte capsules was characterized by scanning electron microscopy (SEM) using MIRA II LMU instrument (Tescan, Czech Republic) at an operating voltage of 20 $\mathrm{kV}$. Size distribution of the carriers was investigated by a set of SEM images in order to obtain a minimum of 100 measurements per sample. Image analysis and statistics were performed using Image J free software. The average size was shown as "mean \pm standard deviation".

The Fe (III) content in magnetic polyelectrolyte capsules was determined relaxometrically through the glass vial test. For this purpose, aliquots of each sample were diluted 1:10 with $\mathrm{HNO}_{3}(70 \% \mathrm{w} / \mathrm{w})$, transferred to glass ampoules and centrifuged for 3 $\mathrm{min}$ at $2000 \mathrm{rpm}$. The glass vials were sealed and placed at $120^{\circ} \mathrm{C}$ overnight to mineralize the samples. Then, the longitudinal relaxation rate $\left(R_{10 b s}\right)$ was measured at $21.5 \mathrm{MHz}(0.5 \mathrm{~T})$ and $25^{\circ} \mathrm{C}$ using a Stelar Spinmaster spectrometer (Stelar srl, Italy). The temperature was controlled by a Stelar VTC-91 airflow heater (Stelar srl, Italy), equipped with a copper-constantan thermocouple (uncertainty was \pm $0.1^{\circ} \mathrm{C}$ ). The millimolar concentration of $\mathrm{Fe}(\mathrm{III})$ was determined as following:

$$
\left[F e^{3+}\right]=\frac{R_{1 o b s}-R_{1 d i a}}{18.47} \times 10(1)
$$

where $R_{1 \text { dia }}\left(R_{1 \text { dia }}=0.481 \mathrm{~s}^{-1}\right)$ is the diamagnetic contribution in acidic conditions, while $18.47 \mathrm{mM}^{-1} \mathrm{~s}^{-1}$ is the relaxivity $\left(r_{1}\right)$ of the Fe (III) aqua ion in acidic conditions at $21.5 \mathrm{MHz}$ and $25^{\circ} \mathrm{C}$ (a value determined by using standard $\mathrm{FeCl}_{3}$ solutions whose concentrations were measured by inductively coupled plasma mass spectrometry (ICP-MS), accuracy \pm $0.1 \%)$.

\section{Relaxivity measurements}

Nuclear magnetic relaxation dispersion (NMRD) profiles of the control (pure) polyelectrolyte submicron capsules and MNP-doped ones (sample S) were recorded at 25 and $37^{\circ} \mathrm{C}$ using a Stelar Fast Field Cycling FFC2000 NMR relaxometer (Stelar srl, Italy) operating at a magnetic field strength ranging from
0.01 to $20 \mathrm{MHz}\left(2.4 \times 10^{-4}\right.$ to $\left.0.47 \mathrm{~T}\right)$. The additional points were measured in the range of $20-70 \mathrm{MHz}$ (0.47-1.65 T) using a tunable magnet (Stelar srl, Italy). The uncertainty of the measurements was lower than $5 \%$.

The longitudinal relaxation rates of control (pure) polyelectrolyte capsule suspension in water measured at various proton Larmor frequencies were equal to water. Therefore, the polyelectrolyte shell did not affect the relaxivity of MNP-doped capsules and their relaxivity changes are caused only by the amount and disposition of magnetite nanoparticles in their structure. Thus, the normalized (to $\mathrm{mM}$ of Fe(III) ions) longitudinal relaxivity $\left(\mathrm{r}_{1}\right)$ of magnetic submicron capsules was calculated according to the following equation:

$$
\mathrm{r}_{1}=\frac{R_{1 o b s}-R_{1 \text { contr }}}{\left[F e^{3+}\right]}(2)
$$

where $R_{1 c o n t r}$ is the diamagnetic contribution of pure water $\left(R_{1 \text { contr }}=0.38 \mathrm{~s}^{-1}\right.$ at $21.5 \mathrm{MHz}$ and $\left.25^{\circ} \mathrm{C}\right)$ [58].

Transverse relaxation rates $\left(R_{20 b s}\right)$ of the obtained samples' suspensions were measured at $21.5 \mathrm{MHz}(0.5$ T) and $25^{\circ} \mathrm{C}$ using a Stelar Spinmaster spectrometer (Stelar srl, Italy), the normalized (to $\mathrm{mM}$ of $\mathrm{Fe}(\mathrm{III})$ ions) transverse relaxivities $\left(\mathrm{r}_{2}\right)$ were calculated then by subtracting the diamagnetic contribution of pure water from these values and dividing by the sample concentration.

Longitudinal and transverse relaxivity were also measured at $300 \mathrm{MHz}(7 \mathrm{~T})$, with a Bruker Avance 300 spectrometer (Bruker Biospin, Germany) equipped with a Micro 2.5 microimaging probe. The samples were transferred into glass capillaries and inserted into an agar phantom. $T_{1}$ and $T_{2}$ relaxation times were then measured. $\mathrm{T}_{1}$ measurement was performed with a Multi Slice Multi Echo (MSME) sequence varying the repetition time from 50 to $8000 \mathrm{~ms}$ (Echo Time (TE)/ Repetition Time (TR)/ Number of Averages (NAV) 3.74/50-8000/10, Matrix 128x128). $\mathrm{T}_{2}$ measurement of the samples was performed with a MSME sequence varying the echo time from 14 to 280 ms (TE/TR/NR 14-280/2000/1, Matrix 256×256).

\section{Sedimentation measurements}

Mobility of the obtained capsules in an external non-uniform magnetic field was measured by photosedimentometry [47]. The rate of capsules' sedimentation induced by a permanent neodymium magnet $(340 \mathrm{mT})$ was measured using the previously described setup [57]. The suspension of capsules in water was poured in the transparent cuvette, and 660 $\mathrm{nm}$ semiconductor laser beam was placed in parallel to the air/water interface. The magnet was applied to the cuvette's wall, so the magnetic field $(326 \mathrm{mT}$ at the cuvette's wall) was directed perpendicularly to the 
laser path. Thus, the time dependencies of the capsule suspension transparency under permanent magnet were obtained.

\section{Cellular experiments}

\section{Cells and Culture Conditions}

Murine macrophage (RAW 264.7) and mammary adenocarcinoma (TS/A) cell lines were used in experiments. RAW 264.7 cells were purchased from American Type Culture Collection (ATCC LGC Standards, Sesto San Giovanni, Italy) and cultured in DMEM supplemented with $10 \%$ (v/v) of FBS, $2 \mathrm{mM}$ L-glutamine, $100 \mathrm{U} / \mathrm{mL}$ penicillin and $100 \mu \mathrm{g} / \mathrm{mL}$ streptomycin at $37{ }^{\circ} \mathrm{C}$ in a humidified atmosphere with $5 \% \mathrm{CO}_{2}$. TS/A cells, derived from a spontaneous BALB/c mammary tumor, were cultured in RPMI-1640 supplemented with $10 \%$ (v/v) of FBS, 2 $\mathrm{mM}$ L-glutamine, $100 \mathrm{U} / \mathrm{mL}$ penicillin and 100 $\mu \mathrm{g} / \mathrm{mL}$ streptomycin at $37{ }^{\circ} \mathrm{C}$ in a humidified atmosphere with $5 \% \mathrm{CO}_{2}$ [59].

\section{In vitro MRI}

MRI of macrophages after their incubation with the obtained magnetic polyelectrolyte capsules was carried out in order to check their ability to be labelled with the proposed system. To this purpose, $1 \times 10^{6}$ RAW 264.7 cells were seeded into $60 \mathrm{~mm}$ Petri dishes. The day after the cells were incubated for 2 hours with MNP-doped capsules (samples $S, C_{1} S, C_{2} S, C_{6} S$ ) at the concentration of $9 \times 10^{7}$ capsules/mL in $2 \mathrm{~mL}$ of medium. The numbers of cells in suspensions, as well as the number of capsules in the samples, were counted using a hemocytometer. Incubation with medium was used as control. At the end of the incubation, cells were profusely washed to remove unbound particles, detached by scraping, resuspended in $10 \mathrm{~mL}$ of PBS and centrifuged twice at $1100 \mathrm{rpm}$ for $5 \mathrm{~min}$. Further, the cells were resuspended in $50 \mu \mathrm{L}$ of PBS, transferred into glass capillaries and centrifuged at $700 \mathrm{rpm}$ for $10 \mathrm{~min}$ to obtain a cell pellet. The capillaries were then inserted into an agar phantom and imaged at $300 \mathrm{MHz}(7 \mathrm{~T})$ with a Bruker Avance 300 spectrometer (Bruker Biospin, Germany) equipped with a Micro 2.5 microimaging probe. $T_{1}$ weighted $\left(T_{1 w}\right)$ Multi Slice Multi Echo MSME (Echo Time (TE)/ Repetition Time (TR)/ Number of Averages (NAV) 3.7/200/24, Matrix 128x128) and $\mathrm{T}_{2 \mathrm{w}}$ RARE (TE/Effective TE/TR/NAV/ Rare Factor (RF) 3.9/31/4000/6/16, Matrix 128x128) sequences were acquired with an axial geometry (3 slices, slice thickness $0.7 \mathrm{~mm}$ ).

The same experiment was performed for selected samples on breast cancer TS/A cells in order to simulate the process of capsules' uptake in tumor. For this purpose, $1 \times 10^{6} \mathrm{TS} / \mathrm{A}$ cells were seeded into 60 mm Petri dishes. The day after the cells were incubated overnight ( 20 hours) or for 1 hour (the timeframe of magnet application planned for in vivo experiments) with MNP-doped capsules (samples $\mathrm{C}_{1} \mathrm{~S}$ and $\mathrm{C}_{6} \mathrm{~S}$ ) at the concentration of $9 \times 10^{7}$ capsules $/ \mathrm{mL}$ in $2 \mathrm{~mL}$ of medium. At the end of the incubation, cells were also washed out from the unbound particles, detached with trypsin/EDTA, resuspended in $10 \mathrm{~mL}$ PBS and centrifuged twice at $1100 \mathrm{rpm}$ for $5 \mathrm{~min}$. The following procedure was the same as described above for RAW 264.7 cells.

Immediately after imaging, the cell pellets (both RAW 264.7 and TS/A) were recollected, resuspended in $200 \mu \mathrm{l}$ of PBS and sonicated in ice with a Bandelin Sonopuls UW2070 probe sonicator (Bandelin, Germany) at $20 \mathrm{~W}$ for $30 \mathrm{~s}$. The protein concentration in each sample was then measured spectrophotometrically using the Bradford protein assay and Jenway $6715 \mathrm{UV}-\mathrm{Vis}$ spectrophotometer (Jenway, USA). To quantify the amount of iron in each sample, cell extracts were digested with concentrated $\mathrm{HNO}_{3}(70 \% \mathrm{w} / \mathrm{w}, 1 \mathrm{~mL})$ under microwave heating using a MicroSYNTH labstation (Milestone, Italy), recollected and analyzed by ICP-MS (Element-2, Thermo-Finnigan, Italy). The mean Fe(III) content per $\mathrm{mg}$ of protein was then calculated for each sample.

\section{Cytotoxicity}

Cytotoxicity measurements were performed for RAW 264.7 cell line to estimate the influence of the obtained MNP-doped capsules on the healthy cells. For the quantification of live and necrotic cells after their incubation with capsules' suspension, $2 \times 10^{5}$ cells were placed on the $60-\mathrm{mm}$ Petri dish and incubated overnight. Then a different number of MNP-doped carriers $\left(9,45\right.$, and $90 \times 10^{6}$ capsules) in $2 \mathrm{~mL}$ of growth medium was added to cells and incubated for 2 hours to simulate the conditions of capsule injection in the bloodstream. The largest number of capsules (90×106) added to cells was chosen the same as injected further to an average mouse (weighing $20 \mathrm{~g}$ ) in vivo. The positive control contained RAW 264.7 cells without any additional treatment, the negative control was prepared by adding $30 \% \mathrm{v} / \mathrm{v}$ of ethanol to the macrophages. The cells of all the groups were detached then and stained with $0.125 \mu \mathrm{g} / \mathrm{mL}$ Acridine orange (AO) and $15 \mu \mathrm{g} / \mathrm{mL}$ Propidium iodide (PI) for $15 \mathrm{~min}$. Finally, the macrophages were triple washed with PBS and measured using an imaging flow cytometer Amnis Mk II (Luminex, USA).

The analysis of the obtained data was performed using the IDEAS software (Luminex, USA). PI-only positive cells were recognized as necrotic ones. AO-positive and double-positive cells were determined as viable cells, where double-positive 
staining was caused by the adsorption of nucleic acids from the surrounded dead cells and their debris on the surface of the viable ones. The data on RAW 264.7 cell viability after their incubation with MNP-doped capsules were represented as "mean \pm standard deviations" $(n=5)$. The one-way analysis of variance (ANOVA) was used to determine the statistical significance of differences in the obtained values. Calculations were made with Microsoft Excel software.

\section{In vivo experiments}

\section{Tumor animal model}

The animal study was approved by the Italian Ministry of Health, the following procedures were in accordance with institutional guidelines and ensured the humane care of the animals. The mice (aged 8 weeks and weighting 18-22 g) were obtained from the animal facility at the Molecular Biotechnology Center of the University of Turin. A xenograft breast tumor mouse model was prepared by subcutaneous injection of $2.5 \times 10^{5}$ adenocarcinoma TS/A tumor cells into the flank of BALB/c female mice. Ten days after tumor implantation, when tumor size reached a diameter of 3-6 $\mathrm{mm}$, the animals were enrolled in the in vivo imaging studies.

\section{MRI experiments}

MRI in vivo experiments were performed at 40 MHz (1 T) with an Aspect M2 High-Performance MRI System (Aspect Magnet Technologies Ltd, Israel) consisting of a NdFeB magnet, equipped with a solenoid $\mathrm{Tx} / \mathrm{Tr}$ coil of $35 \mathrm{~mm}$ inner diameter. This system was equipped with fast gradient coils (gradient strength of $450 \mathrm{mT} \mathrm{m}^{-1}$ at $60 \mathrm{~A}$, ramp time of $250 \mu$ s at $160 \mathrm{~V}$ ) with a field homogeneity of $0.2-0.5$ gauss. Before MRI experiments, the animals were anesthetized by intramuscular injection of tiletaminezolazepam (Zoletil 100; Virbac, Milan, Italy) $20 \mathrm{mg}$ $\mathrm{kg}^{-1}$ and xylazine (Rompun; Bayer, Milan, Italy) $5 \mathrm{mg} \mathrm{kg}^{-1}$.

The suspension of magnetic polyelectrolyte capsules was diluted in HEPES/ $\mathrm{NaCl}$ buffer (to obtain a suspension 280 mOsm, pH 7.4) and injected into the tail vein. The mice underwent MRI before and at different time points $(1,4.5,24$ and 48 hours) after the capsule injection. A standard $\mathrm{T}_{2}$ Fast Spin Echo sequence was used with the following parameters: TR/TE/NEX 2500/49/4, resolution of $250 \mu \mathrm{m}$, slice thickness of $1.5 \mathrm{~mm}$.

Image analysis and statistics were performed using Image J free software. The mean signal intensity (SI) values were calculated on a region of interest
(ROI). The measured SI was normalized to a water-containing reference tube to take into account the differences in the absolute signal intensity values $\left(S I_{n}\right.$, where n means "normalized") among different images obtained after mouse repositioning in the MRI scanners. The normalization was carried out by dividing the SI values of the ROI drawn on the organ of interest to the $S I$ values of the ROI drawn inside the reference tube $\left(S I_{\text {ref }}\right)$ :

$$
S I_{n}=\frac{S I}{S I_{\text {ref }}}(3)
$$

The mean percent of signal change (SC) was calculated according to the following equation:

$$
S C=\frac{S I_{n} \text { post }-S I_{n} \text { pre }}{S I_{n} \text { pre }} \times 100 \%(4)
$$

where $S I_{n \text { pre }}$ - the mean signal intensity of the organ of interest before the capsule injection, $S I_{\text {post }}$ - the mean signal intensity of the organ of interest after the capsule injection.

The data were calculated from six independent experiments, unless otherwise stated, and expressed as the "mean \pm SE". ANOVA and the $t$-test were performed to determine the statistical significance of differences in SE in tumor between different time points for the same experimental group $(p<0.05)$ and between different experimental groups $(p<0.01)$.

\section{Magnetic targeting}

Active magnetic addressing of MNP-doped capsules was performed by the application of a permanent magnet with a concentrator $(0.5 \mathrm{~T})$ to the tumor. First, the magnet was placed on the tumor, and then the contrast agent was injected into the tail vein (Figure 1). The magnet was kept applied to the tumor for 1 hour.

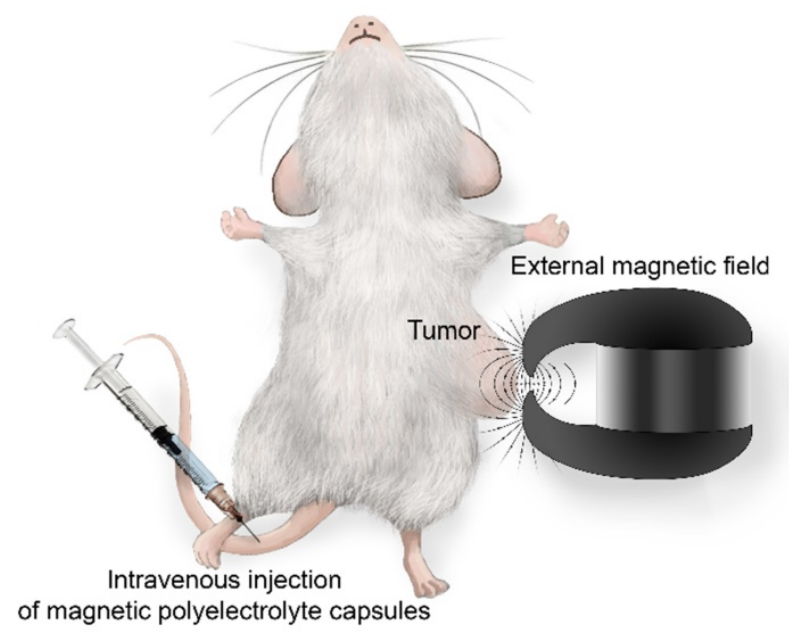

Figure 1. Schematic illustration of the magnetic targeting for MNP-doped capsules. 


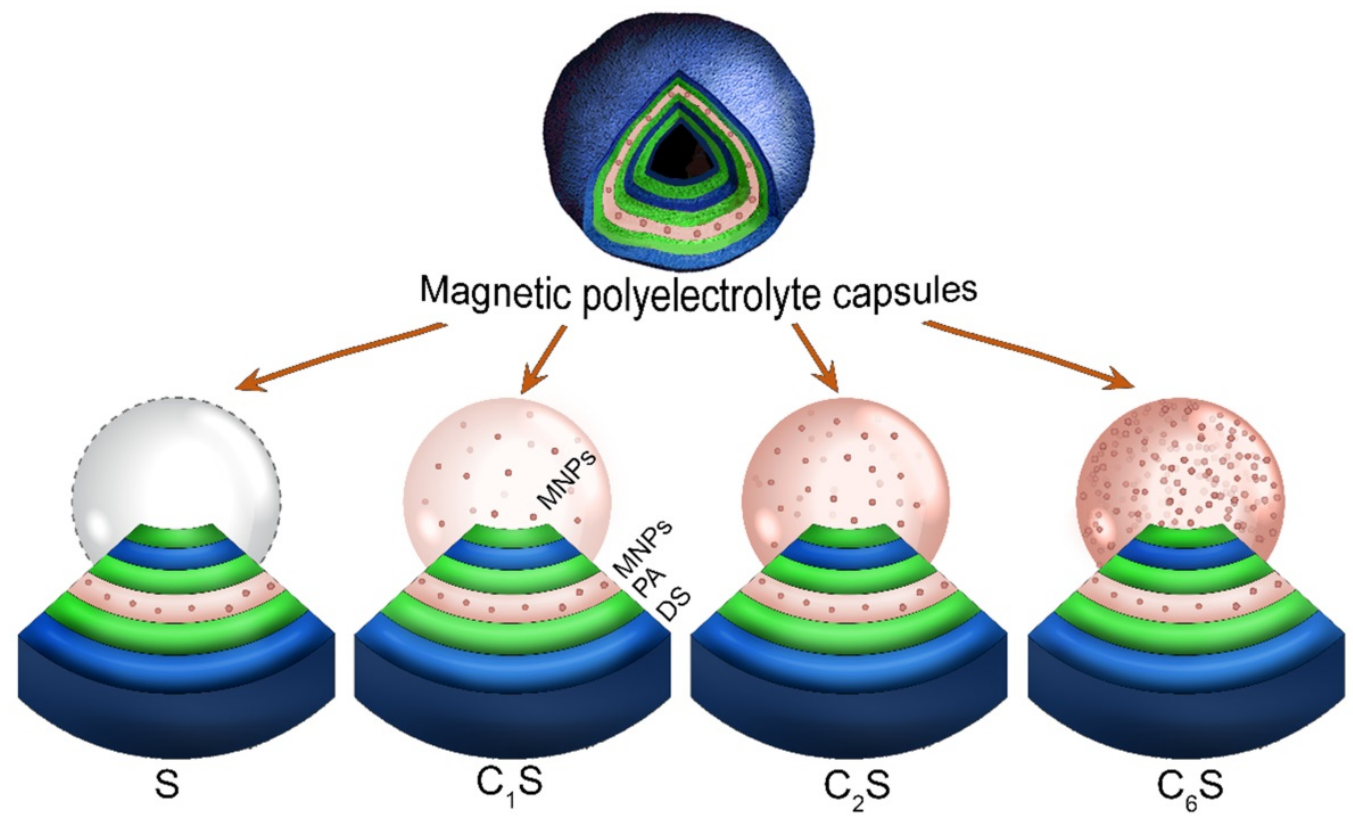

Figure 2. Scheme representing the structure of the obtained magnetic polyelectrolyte capsules: sample S containing magnetic nanoparticles (MNPs) in the capsules' shell and samples $\mathrm{C}_{n} \mathrm{~S}$ containing MNPs in both, the shell and the inner volume of the capsules ( $\mathrm{n}$ is the number of MNPs loading cycles). All the sample types contain poly-arginine (PA) and dextran sulfate (DS) polymers in their shell.

\section{Histology}

For ex-vivo histological studies, tumor, liver and spleen were excised at different time points post injection and fixed overnight in a $10 \% \mathrm{NBF}$, dehydrated and embedded into paraffin. Then tissue slices ( $5 \mu \mathrm{m}$ of thickness) were cut with a microtome. The presence and localization of iron was investigated by means of Perls' Prussian blue staining. Briefly, sections were hydrated, covered with equal parts mixture of $5 \%$ potassium ferrocyanide and $5 \% \mathrm{HCl}$ for 1 hour, washed in distilled water and counterstained with NFR for 10 minutes. Finally, sections were dehydrated, cleared, mounted and examined under an Olympus BX14 microscope (Olympus, USA).

\section{Results and Discussion}

\section{In vitro characterization of magnetic polyelectrolyte capsules}

Layer-by-layer (LbL) assembled polyelectrolyte capsules represent a unique tool to fabricate micronand submicron-sized theranostic systems which are capable of targeted delivery and controlled in-situ release [41, 60-64]. So far, effective encapsulation of different drugs, including anticancer [65-68], RNA/DNA [69-71], growth factors [72, 73], antigens [74-76], and other bioactive substances [77-81] was demonstrated by means of polyelectrolyte capsules. Various multifunctional imaging agents were successfully fabricated using LbL technique as well [82-84]. Embedding of magnetite nanoparticles into the structure of polyelectrolyte capsules opens up the perspective for their application in MRI [44, 85-88] as well as for improving drug delivery and controlled drug release [27, 41, 42, 44].

Considering this, magnetic nanoparticle (MNP)-doped polyelectrolyte capsules of different structure were formed and tested for capability of their delivery to tumor and MRI-visualization.

To start with, the colloid of MNPs was synthesized by the chemical precipitation method in an inert atmosphere [89, 90]. A TEM image of the obtained nanoparticles is presented in Figure SM1 (Supplementary Material, SM). The image demonstrates a spherical shape of MNPs with an average size of $5.0 \pm 0.9 \mathrm{~nm}$. DLS measurements show a particle hydrodynamic diameter of $8.8 \pm 2.0 \mathrm{~nm}$ (Figure SM1 in Supplementary Material).

Magnetic polyelectrolyte capsules were then formed using this MNP suspension. Different capsule structures were formulated in order to determine a balanced carrier composition in terms of magnetic and MRI contrast properties. To accomplish this, MNPs were embedded either into the capsules' shell (sample S) or into both, the shell and the inner volume of the capsules (samples CnS, where $\mathrm{n}$ is the number of MNPs loading cycles). The scheme representing the structure of the obtained capsules is provided in Figure 2. The iron content in the obtained capsules (determined as Fe(III)) depending on their structure is presented in Table 1.

Biodegradability is a crucial property of drug delivery systems. For micron- and submicron-sized capsule formulation, the biodegradable polymers, 
either synthetic or natural, are often used since they are capable of being split into biocompatible products by chemical or enzyme-catalyzed hydrolysis [91]. Poly-L-arginine (PA) and dextran sulfate (DS) have been widely employed to design polyelectrolyte capsules that were found to be biocompatible and degradable both in vitro and in vivo $[92,93]$. Thus, here the MNP-doped capsules were fabricated using these polymers by LbL technique. SEM images of the obtained carriers are presented in Figure 3. The average size of the capsules was $0.8 \pm 0.1 \mu \mathrm{m}$.

The SEM images clearly demonstrated that the morphology of the capsules' surface differed depending on the amount of incorporated magnetite. In particular, sample $S$ containing MNPs only in polyelectrolyte shell represented thin submicron capsules with quite smooth surface (Figure 3 a, e). Embedding of MNPs into the inner volume of the capsules resulted in increased wall thickness (Figure 3 b-d and f-h). Moreover, the higher magnetite content was in capsules, the rougher and thicker they appeared.

According to our previous data [46], the concentration of MNPs adsorbed at the shell defines MRI contrast properties of polyelectrolyte capsules. Specifically, the MR contrast for both $T_{1}$ and $T_{2}$ was shown to depend on the distance between magnetite nanoparticles in the capsules' shell. With the larger distance between MNPs in the shell layer, relaxation rate increases resulting in MR signal enhancement. In the current study, the amount of magnetite in a shell was chosen to provide the best MRI contrast [46]. However, the polyelectrolyte carriers containing MNPs only in their shell possessed low sensitivity to magnetic field due to insufficient magnetite concentration [47]. Meanwhile, the embedding of higher amount of MNPs into the structure of polyelectrolyte capsules can open up the possibilities for their navigation with external magnetic field [42, $44,94,95]$.

The opportunity to improve the magnetic navigation property of polyelectrolyte capsules by the incorporation of MNPs into their inner volume was studied here. The effect of changing the amount of incorporated magnetite on the mobility of the obtained capsules in an external magnetic field was measured by means of photosedimentometry. The results are represented in Figure 4. The graphs demonstrate the change in transmission of the capsule suspensions under the application of a magnet depending on the capsules' structure. The photographs of the capsule suspensions in cuvette before and after the magnet application are presented in Figure SM2 in Supplementary Material.

Table 1. Structure and characteristics of the magnetic polyelectrolyte capsules at $0.5 \mathrm{~T}$ and $25^{\circ} \mathrm{C}$

\begin{tabular}{|c|c|c|c|c|c|}
\hline Sample & Structure & {$\left[\mathrm{Fe}^{3+}\right] \mathrm{mM}$} & $\mathbf{r}_{1}(\mathrm{mM} \times \mathrm{s})^{-1}$ & $\mathrm{r}_{2}(\mathrm{mM} \times \mathrm{s})^{-1}$ & $\mathbf{r}_{2} / \mathbf{r}_{1}$ \\
\hline$S$ & (PA/DS/PA/MNPs/PA/DS) & 0.43 & 19.7 & 92.1 & 4.7 \\
\hline$C_{1} S$ & (MNPs)/(PA/DS/PA/MNPs/PA/DS) & 4.4 & 17.8 & 79.3 & 4.5 \\
\hline $\mathrm{C}_{2} \mathrm{~S}$ & $(\mathbf{M N P s})_{2} /(\mathrm{PA} / \mathrm{DS} / \mathrm{PA} / \mathbf{M N P s} / \mathrm{PA} / \mathrm{DS})$ & 8.8 & 5.3 & 23.4 & 4.4 \\
\hline$C_{6} S$ & (MNPs)6/(PA/DS/PA/MNPs/PA/DS) & 15.8 & 0.6 & 1.5 & 2.7 \\
\hline
\end{tabular}
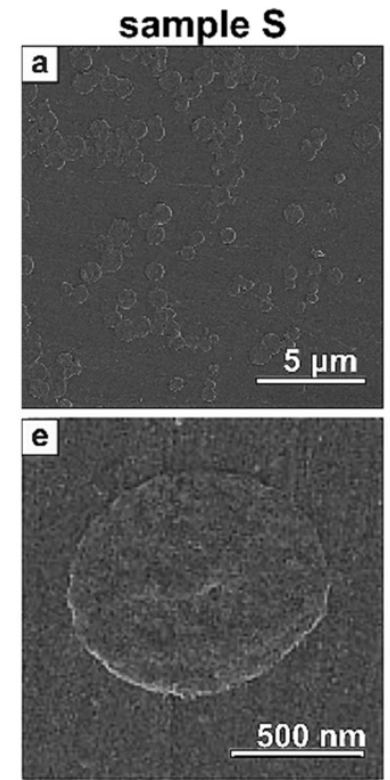
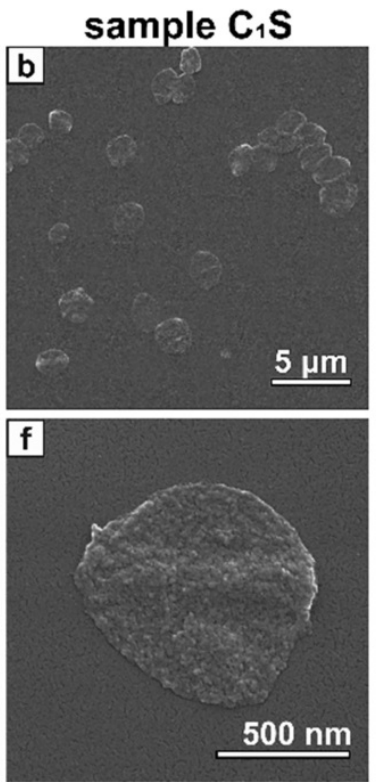
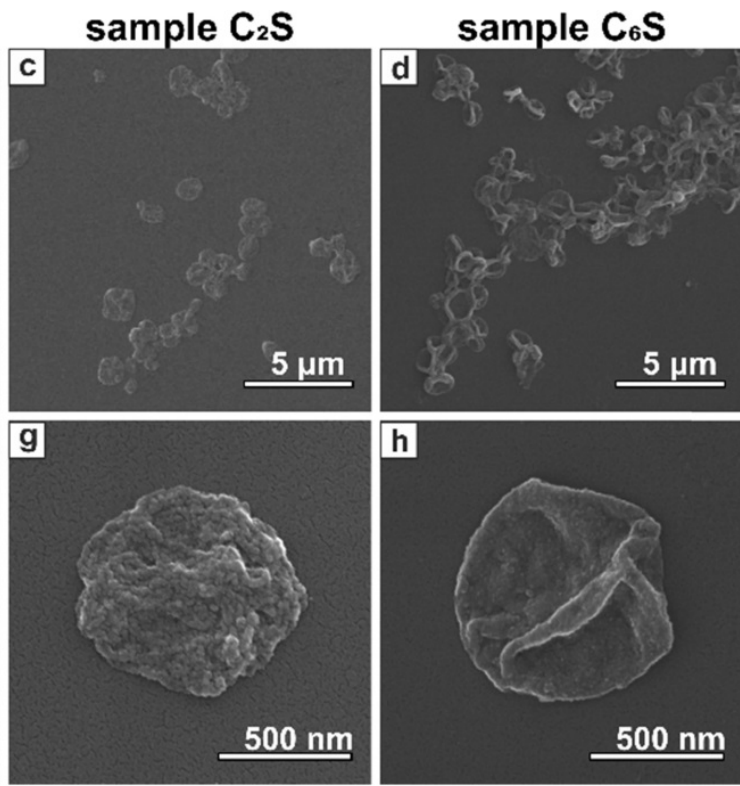

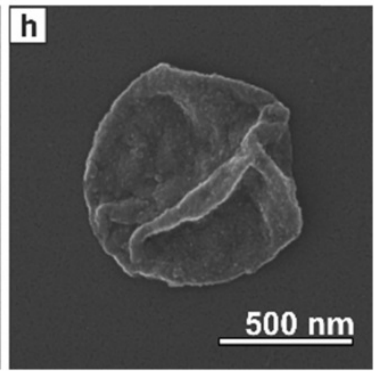

Figure 3. SEM images of magnetic polyelectrolyte capsules: (a, e) - sample $S$; (b, f) - sample $C_{1} S$; (c, g) - sample $C_{2} S$; (d, h) - sample $C_{6} S$ performed at different magnifications. 


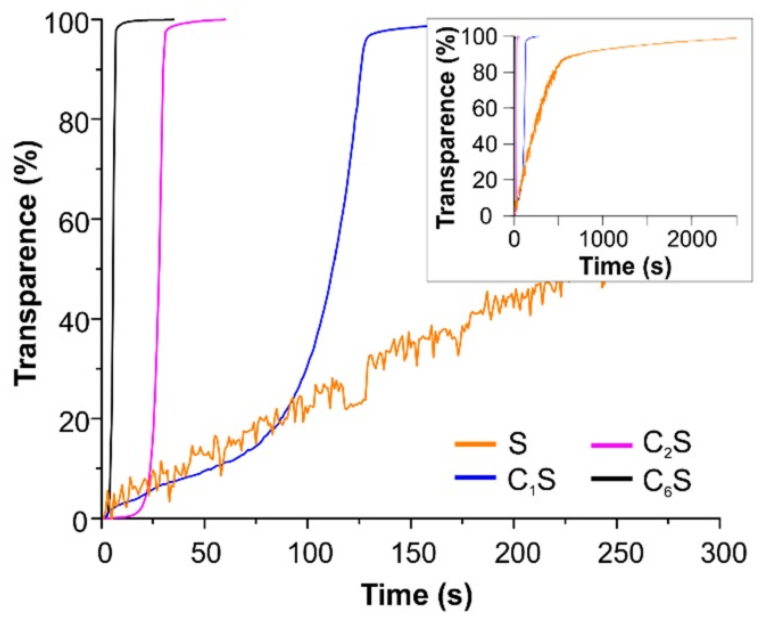

Figure 4. Photosedimentometry of magnetic polyelectrolyte capsules $S, C_{1} S, C_{2} S$ and $\mathrm{C}_{6} \mathrm{~S}$ dispersed in water during the time represented as time dependence of the suspension transparence in an external magnetic field.

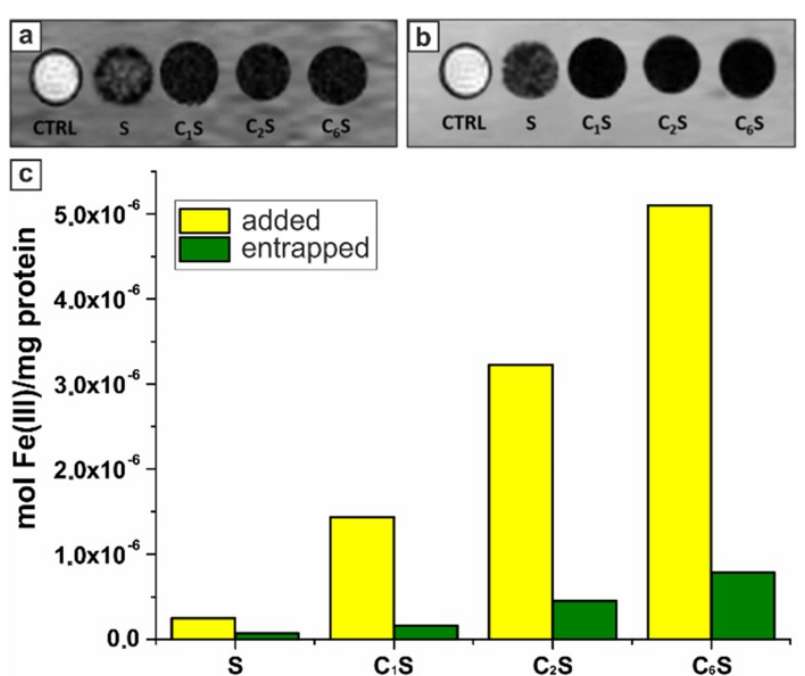

Figure 5. $T_{1} w$ (a) and $T_{2} w$ (b) magnetic resonance images acquired at $7 \mathrm{~T}$ of glass capillaries containing RAW 264.7 cells incubated for 2 hours in the absence (control cells, CTRL) and in the presence of magnetic polyelectrolyte capsules (samples $\mathrm{S}, \mathrm{C}_{1} \mathrm{~S}$, $\mathrm{C}_{2} \mathrm{~S}, \mathrm{C}_{6} \mathrm{~S}$ ). (c) Histogram representing the amount of iron added to (yellow columns) and entrapped by (green columns) RAW 264.7 cells after the 2-hours incubation with samples $\mathrm{S}, \mathrm{C}_{1} \mathrm{~S}, \mathrm{C}_{2} \mathrm{~S}$, and $\mathrm{C}_{6} \mathrm{~S}$ represented as moles of iron per $1 \mathrm{mg}$ of cellular proteins; the calculations were based on ICP-MS analysis of $\mathrm{Fe}(\mathrm{III})$ content and spectrophotometric evaluation of proteins by means of Bradford assay.

According to the obtained data, the sample $\mathrm{C}_{6} \mathrm{~S}$ was the most sensitive to an external non-uniform magnetic field. However, it possessed the lowest $r_{1}$ and $\mathrm{r}_{2}$ relaxivities at $21.5 \mathrm{MHz}(0.5 \mathrm{~T})$ (Table 1). In contrast, the sample $S$ without magnetite in the inner volume revealed as the best $T_{2}$ contrast agent among all studied carriers with an $\mathrm{r}_{2} / \mathrm{r}_{1}$ ratio of 4.7 . The $\mathrm{T}_{1}$-NMRD profile for this sample (at 25 and $37^{\circ} \mathrm{C}$ ) is shown in Figure SM3, and it is characterized by a broad hump centered at around $10 \mathrm{MHz}$. It should be noted that the obtained profile appears very similar to Endorem $^{\mathrm{TM}}$, a dextran-coated iron oxide NPs formulation formerly approved for clinical MRI, and to other iron-oxide loaded nanosystems $[53,96]$, thus suggesting similar relaxometric properties for these magnetic systems.

A decreasing tendency was observed at 21.5 $\mathrm{MHz}$ (Table 1, Figure SM4) for both $\mathrm{r}_{1}$ and $\mathrm{r}_{2}$ relaxivities with the enhancement of magnetite content inside the capsule, with $\mathrm{r}_{2}$ values displaying a sharper decay. A similar trend has been previously reported for magnetic polymer micro- and submicron capsules [88,97], where it was ascribed to the effect of clustering and inhomogeneous spatial distribution of the embedded MNPs within the capsules on the dipolar magnetic energy and on the water protons/MNP interaction. Interestingly, despite these $r_{1}$ and $r_{2}$ decays, their ratio $\left(r_{2} / r_{1}\right)$ remained almost the same with increasing loading cycles up to 2 (samples $\mathrm{S}, \mathrm{C}_{1} \mathrm{~S}$ and $\mathrm{C}_{2} \mathrm{~S}$ ). Meanwhile, with the more drastic increase of magnetite content inside the capsule, as for the $\mathrm{C}_{6} \mathrm{~S}$ sample, the possibility of MNP aggregation raised, leading to a significant $r_{2} / r_{1}$ decrease (see Table 1).

As far as the proposed contrast agent is capsule-based and the amount of MNPs inside these capsules differed depending on the sample structure, it was useful to compare also the relaxivities of the samples $\mathrm{S}$ and $\mathrm{C}_{6} \mathrm{~S}$ normalizing them to the capsules' concentration (indicated as $r_{1 \text { caps }}$ and $r_{2 \text { caps }}$ ) instead of concentration of $\mathrm{Fe}(\mathrm{III})$ ions. Such comparison demonstrated no drastic difference between the obtained $\mathrm{r}_{1 \text { caps }}$ values $\left(9.3 \mathrm{vs} 9.9(\mathrm{pM} \times \mathrm{s})^{-1}\right.$ for the $S$ and $\mathrm{C}_{6} \mathrm{~S}$ samples, respectively). However, the transverse relaxivity $\mathrm{r}_{2 \text { caps }}$ for the sample $S$ was still higher than for the sample $\mathrm{C}_{6} \mathrm{~S}$ (43 vs $26(\mathrm{pM} \times \mathrm{s})^{-1}$, respectively).

Before carrying out the in vivo study, the possibility to generate a sufficient MRI contrast was assessed for the RAW 264.7 cells labeled with the synthesized magnetic polyelectrolyte capsules. This murine phagocytic cell line was used as an immune cell model simulating capsules' uptake [98]. $\mathrm{T}_{1}$ and $\mathrm{T}_{2}$-weighted $\mathrm{MR}$ images were acquired at $7 \mathrm{~T}$ on cellular pellets obtained upon 2-hours incubation with MNP-doped capsules (samples $S, C_{1} S, C_{2} S, C_{6} S$ ). In order to study the effect of the sample structure, the concentration of capsules added to cells was set at the same level for all the samples, while the total amount of incubated iron differed. The resulting $\mathrm{T}_{1^{-}}$and $\mathrm{T}_{2}$-weighted MR images are presented in Figure $5(\mathrm{a}$, b).

The MNP-doped polyelectrolyte capsules demonstrated the ability to be entrapped by macrophages generating sufficient MRI contrast in both $T_{1}$ and $T_{2}$ weighted images. The $R_{1 \text { obs }}$ and $R_{2 o b s}$ relaxation rates were not measurable for the samples $\mathrm{C}_{1} \mathrm{~S}, \mathrm{C}_{2} \mathrm{~S}$ and $\mathrm{C}_{6} \mathrm{~S}$ due to the extremely high $\mathrm{Fe}(\mathrm{III})$ content. High iron concentration resulted in a strong $\mathrm{T}_{2}$ effect, affecting also $\mathrm{T}_{1} \mathrm{~W}$ contrast as decreasing $\mathrm{T}_{1}$ signal intensity. While for the sample $S$, where the 
magnetite content was significantly lower, the $\mathrm{T}_{1}$ and $\mathrm{T}_{2}$ signal was much higher than for $\mathrm{CnS}$ ones. Thus, the $r_{1}$ and $r_{2}$ relaxivities (at $7 \mathrm{~T}$ and normalized to $\mathrm{Fe}(\mathrm{III})$ ions) were calculated just for the cells incubated with sample $S$ and corresponded to 11.5 and $711.5 \mathrm{mM}^{-1} \mathrm{~s}^{-1}$, respectively. It should be noted that $R_{10 b s}$ and $R_{2 o b s}$ values were more than 100 and 5 times higher than the relaxation rates measured for the control RAW 264.7 cells (incubated without capsules), respectively ( 29.6 vs $0.3 \mathrm{~s}^{-1}$ for $\mathrm{R}_{1 \mathrm{obs}} ; 92.7 \mathrm{vs}$ 20.6 for $\left.R_{2 o b s}\right)$. The relaxivities of the magnetic polyelectrolyte capsules acquired at $7 \mathrm{~T}$ are provided in Table SM1 (Supplementary Material).

The amount of iron entrapped by cells after their incubation with capsules was evaluated by ICP-MS. The mean Fe(III) content per mg of cellular protein for each sample was then calculated based on these data and presented in Figure 5c. These results demonstrated a good labeling ability in vitro rendering the proposed capsules-based contrast agent promising for in vivo MRI application. According to spectrophotometric evaluation of proteins with a Bradford assay, $1 \mathrm{mg}$ of proteins was equal to $10^{6}$ RAW cells.

High amount of iron was entrapped also when the MNP-doped capsules were incubated with adenocarcinoma TS/A cells simulating the process of capsules' uptake in targeted tumor tissues (see Figure SM5 in SM). The drastic changes in MR properties of these tumor cells resulting from the internalization of the capsules were noted as well (Figure SM5 in SM). Accordingly, one might expect to observe the steep decrease of MR signal in tumor as soon as the proposed theranostic system is successfully delivered there.

Cytotoxicity of the obtained capsules was also determined prior to the start of in vivo experiments.
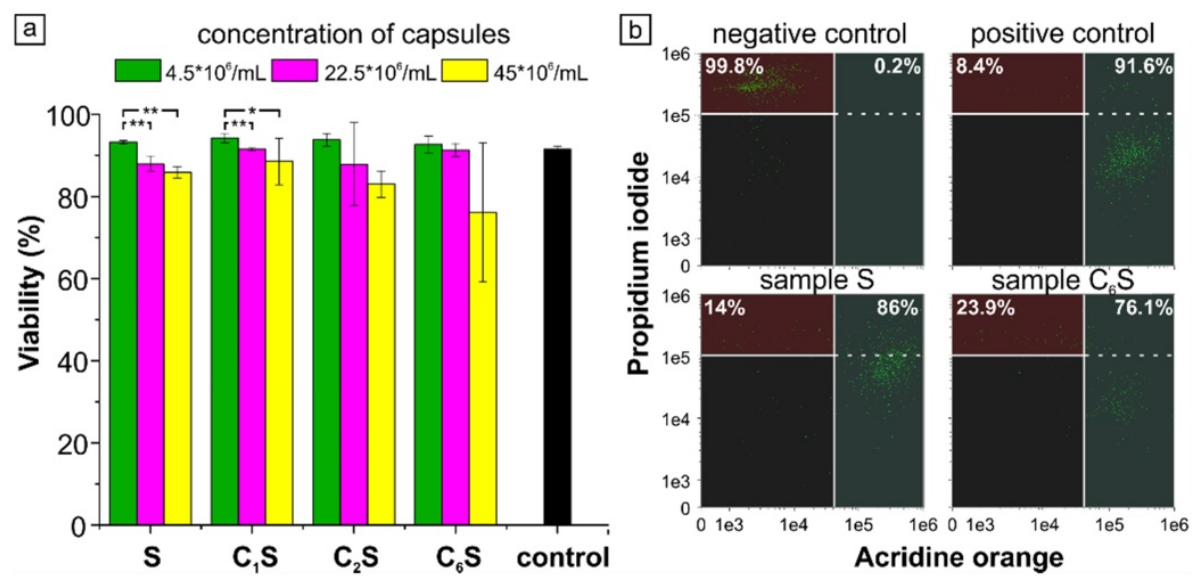

Figure 6. (a) Cytotoxicity of magnetic polyelectrolyte capsules $S, C_{1} S, C_{2} S$ and $C_{6} S$. The statistically significant differences calculated by ANOVA-test are represented as $*(p<0.05)$ and $* *(p<0.01)$. (b) Flow cytometry scatter plot for Acridine orange/Propidium lodide double stained RAW 264.7 cells after capsules' uptake. Negative control represents cells without capsules after induced necrotic death; positive control represents cells without any additional treatment.
For this purpose, MNP-doped capsules of various concentrations were incubated with RAW 264.7 cells. For cytotoxicity assay, the largest number of capsules added to a dish of cells was $9 \times 10^{7}\left(45 \times 10^{6}\right.$ capsules/mL of medium), as it corresponded to the number of capsules further administered to an average mouse (weighing $20 \mathrm{~g}$ ) in vivo. The results are presented in Figure 6.

The study of macrophages viability under the 2-hour incubation with magnetic polyelectrolyte capsules of various structure performed using double AO-PI staining demonstrated a good cytocompatibility of the samples added even at the highest concentration of 450 capsules per cell $\left(9 \times 10^{7}\right.$ capsules per dish). Although cell viability slightly varied with the capsules' structure and concentration, it was no less than $76 \%$ for the $\mathrm{C}_{6} \mathrm{~S}$ sample (Figure 6 , a). For a better illustration, a scatter plots showing the distribution of double stained AO-PI RAW 264.7 cells for the highest concentration of $\mathrm{S}$ and $\mathrm{C}_{6} \mathrm{~S}$ samples were provided (Figure $6 \mathrm{~b}$ ). As far as the viability was higher than $70 \%$ for all the samples, the carriers were considered non-cytotoxic in accordance with ISO 10993-5:2009 [99]. According to ANOVA-test, the change in capsule's structure for the same capsule concentration did not lead to any statistically significant differences in RAW 264.7 cell viability. The change in carrier concentration caused statistically significant difference only for $S$ and $C_{1} S$ samples, while for the others the differences were not statistically significant even if the average values were noticeably lower.

Based on the data obtained, the capsules with MNPs incorporated only into their shells (sample S) were selected as the best $T_{2}$ contrast agent in terms of MRI visualization properties in vitro. Meanwhile, the capsules containing the highest amount of magnetite (MNPs in the shell and inner volume of the capsules, sample $\mathrm{C}_{6} \mathrm{~S}$ ) revealed as the optimal system concerning the highest sensitivity to a magnetic field and possibility to generate a sufficient negative MRI contrast upon the cellular internalization. At the same time, both samples applied even at a high concentration did not cause a prominent cytotoxic effect. By this means, the samples $\mathrm{S}$ and $\mathrm{C}_{6} \mathrm{~S}$ were chosen for the following in vivo assessment. 


\section{Magnetic polyelectrolyte capsules in grafted breast tumor mouse model}

The possibility to deliver the obtained magnetic polyelectrolyte capsules to a tumor was then studied by means of in vivo MRI. To be effective, the proposed theranostic system needs to provide a good balance between MR contrast and magnetic navigation properties when applied in vivo as we aimed at successful magnetic targeting realization.

TS/A cells were used to form a grafted tumor model in mice. It is well known that high permeability of the tumor vasculature compared to normal tissue allows large molecules and small particles to enter the tumor interstitial space and the compromised lymphatic filtration allows them to stay there (EPR effect) [100]. As far as the permeabilized vasculature varies from 200 to $800 \mathrm{~nm}$, such passive targeting effect is expected to take place for the proposed delivery system as well. That should result in intratumor accumulation of the capsules and, thus, in MR contrast enhancement. Assuming this possible effect, the control experiments on MNP-doped capsules' injection not followed by external magnet application (so-called "no magnet") were performed to estimate the real effect of magnetic targeting.

The sample $S$ was tested first as providing better $\mathrm{T}_{2}$ contrast properties. $4 \mathrm{nmol} \mathrm{Fe}(\mathrm{III}) / \mathrm{g}\left(4.5 \times 10^{6}\right.$ capsules/g body weight) were administered to mice $(n=6)$ by intravenous injection. The accumulation in tumor, liver and spleen tissues was assessed 1, 4.5, 24 and $48 \mathrm{~h}$ after the injection by acquiring MR images at $1 \mathrm{~T}$ and calculating $\mathrm{T}_{2}$ contrast. The contrast was expressed as the "percent of $\mathrm{T}_{2}$ signal change" representing the percentage change in the mean signal intensity of the organ of interest in comparison to pre-injection images (see Material and Methods). The results are shown in Figure 7 (a).

Injection of sample $S$ provided generation of a marked MRI contrast in vivo. The maximum of $\mathrm{T}_{2}$ signal decrease in tumor $(-18 \pm 4 \%)$ was registered 1 hour after the injection, meaning that the amount of the MNP-doped capsules in tumor was maximal at this time point. The systemic clearance of not internalized capsules led to a sufficient decrease in $\mathrm{T}_{2}$ signal intensity in liver and spleen as well $(-39 \pm 4 \%$ and $-19 \pm 4 \%$, respectively).
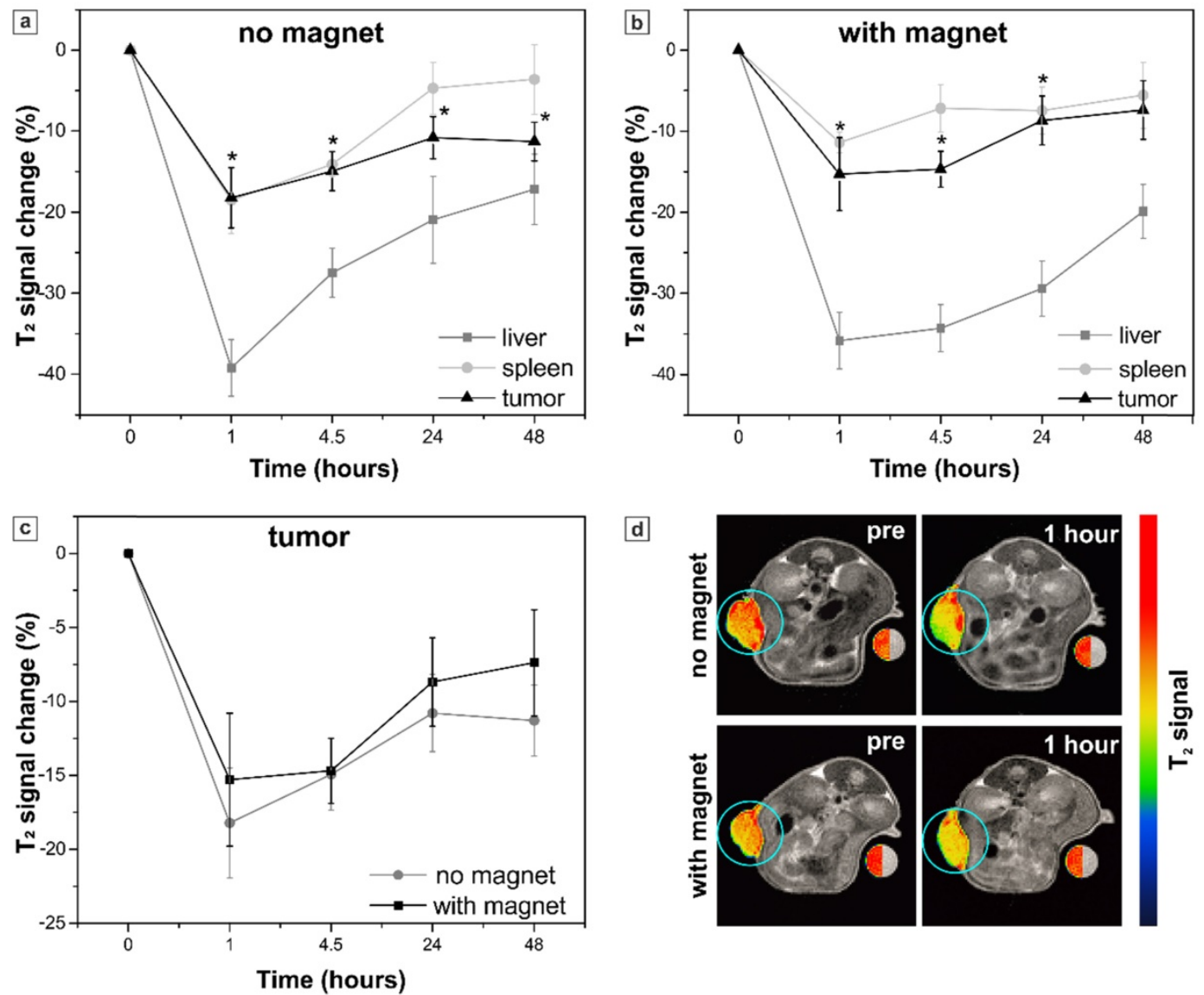

d

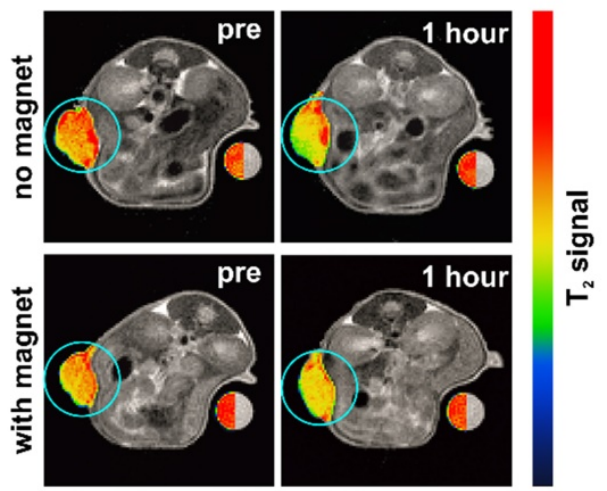

Figure 7. Sample S. Percent of $T_{2}$ signal change in the organs of interest (tumor, liver and spleen) at different time points resulting from the systemic injection of sample $\mathrm{S}$ : (a) without and (b) with the application of the external magnet to the tumor, an asterisk $\left(^{*}\right)$ indicates significant differences in $T_{2}$ signal change in tumor in comparison to pre-injection images; (c) comparison of $T_{2}$ signal change in the mice tumors with and without magnet application. The values correspond to the "mean \pm SE", $n=6$ per each group (no magnet/ with magnet). (d) Representative $T_{2}$-weighted axial MR images of mice acquired before (pre) and 1 hour after the injection. Tumors are marked with blue circles and presented in pseudo colors; a water-containing reference tube is presented in both, original grayscale and pseudo colors. 
In order to improve the tumor accumulation of the proposed submicron capsule-based MR probe, magnetic targeting was applied then. To accomplish this, a permanent magnet with a concentrator $(0.5 \mathrm{~T})$ was placed on the tumor right before the injection of capsules (Figure 1, Materials and Methods) and kept applied for 1 hour further. MR images were acquired again at $1 \mathrm{~T}$ and $\mathrm{T}_{2}$ contrast was measured. The obtained results are presented in Figure 7 (b). The comparison of the mean $\mathrm{T}_{2}$ signal change in the mice tumors with and without magnet application is presented in Figure 7 (c, d).

The maximum of $\mathrm{T}_{2}$ signal decrease in tumor was again reached $1 \mathrm{~h}$ after the injection. However, no significant difference was observed between the groups of mice measured with and without the external magnet application, neither 1 hour after the injection (-15 $\pm 5 \%$ and $-18 \pm 4 \%$, respectively), nor at the later time points (Figure 7c). Quantification of the $\mathrm{T}_{2}$ signal in liver and spleen did not show any significant impact of the magnet application as well. Meanwhile, the axial MR images of mice (Figure 7d) demonstrated the absence of signal artefacts in tumor after the application of the magnet that presumably resulted from a more homogeneous distribution of the magnetic particles. By this means, the exposure to a magnetic field may allow the overcoming of the impaired drugs/nanosystems' distribution often observed in tumors due to disordered vasculature, hypoxic microenvironments and elevated interstitial fluid pressure [40,101].

As mentioned above, the MR contrast enhancement in the tumor indicates a successful targeting of the contrast agent. Thus, polyelectrolyte capsules with MNPs incorporated into their shells (sample S), which played a role of such agent here providing almost $20 \% \mathrm{~T}_{2}$ signal change, appeared to be a promising delivery system. Once co-loaded with an anticancer drug, these capsules may contribute to its accumulation in tumor tissue. Furthermore, gradual fading of $T_{2}$ contrast within 48 hours in both spleen (approaching the pre-injection values) and liver (recovering to half of the pre values) took place in our study (Figure $7 \mathrm{a}, \mathrm{b}$ ). That pointed at a relatively short period of capsules' elimination allowing for repeated administrations in anticancer therapy. Realization of magnetic targeting of polyelectrolyte capsules to the tumor, which has failed for sample $S$ due to its low sensitivity to external magnetic field, might enhance the amount of a delivered drug as well. However, the more sensitive magnetic system should be used for this purpose.

Thus, in the second set of experiments, in vivo MRI was carried out in mice receiving sample $\mathrm{C}_{6} \mathrm{~S}$ as it demonstrated the highest sensitivity to a magnetic field in vitro. Here, $147 \mathrm{nmol} \mathrm{Fe}(\mathrm{III}) / \mathrm{g}$ were intravenously administered to mice $(n=4)$ maintaining the same capsules' concentration used for the sample $\mathrm{S}\left(4.5 \times 10^{6}\right.$ capsules/g body weight). The $\mathrm{T}_{2}$ contrast in tumor, liver and spleen tissues was assessed again 1 , $4.5,24$ and $48 \mathrm{~h}$ after the injection by acquiring MR images at $1 \mathrm{~T}$ and the resulting data are shown in Figure 8.

Injection of the sample $\mathrm{C}_{6} \mathrm{~S}$ resulted in a significant $T_{2}$ signal decrease (in comparison to pre injection values) in tumors of both mice groups, exposed or not to the external magnetic field (Figure 8 $a, b)$. However, no enhancement in MRI contrast was observed in tumor if compared to the treatment with sample $S$, while the mean $T_{2}$ signal in liver and spleen decreased drastically. Such an effect is caused by the dense packing of MNPs in the inner volume of the $\mathrm{C}_{6} \mathrm{~S}$ capsules. As described above, a high amount of magnetite inside the capsule negatively affects the $T_{2}$ contrast properties of the sample (Table 1). Enhanced local concentration of MNPs promotes a strong "quenching" effect on the observed relaxivity of intact capsules [46,102]. That resulted in a less accentuated $\mathrm{T}_{2}$ signal decrease in tumor for the sample $\mathrm{C}_{6} \mathrm{~S}$ as compared with sample S. Meanwhile, upon the degradation of MNP-doped capsules, liberation of magnetite particles together with the enhancement of interparticle distance take place causing a sufficient change in MR contrast of the reticuloendothelial system (RES)-associated organs [46]. Here, it resulted in such a drastic decrease of $\mathrm{T}_{2}$ signal in liver and spleen after the injection of sample $\mathrm{C}_{6} \mathrm{~S}$ in comparison to sample $S$, especially 48 hours post injection. Specifically, in liver the mean $\mathrm{T}_{2}$ signal change values obtained at this time point for sample $\mathrm{C}_{6} \mathrm{~S}$ exceeded the ones obtained for sample $S$ by 3 times. In spleen a more than 10-times higher $T_{2}$ signal decrease and thus, $\mathrm{T}_{2}$ contrast enhancement, was registered 48 hours after the injection of sample $\mathrm{C}_{6} \mathrm{~S}$ (compared again to treatment with sample S).

Nevertheless, the main effect associated with the injection of highly loaded polyelectrolyte/magnetite capsules (sample $\mathrm{C}_{6} \mathrm{~S}$ ) occurs when comparing their injection with and without an external magnetic field application (Figure $8 \mathrm{c}$, d). The capsules have demonstrated a good magnetic sensitivity in vivo providing a significantly stronger $(p<0.01) T_{2}$ contrast 1 hour after the injection in tumor exposed to magnet $\left(-12 \pm 2 \%\right.$ vs $-4 \pm 2 \% T_{2}$ signal change for mice treated and not treated with magnet, respectively). At the same time, significantly lower $(p<0.05) \mathrm{T}_{2}$ contrast was observed in spleen of the treated with magnet mice $\left(\mathrm{T}_{2}\right.$ signal change was $-43 \pm 7 \%$, while for untreated mice it corresponded to $-65 \pm 5 \%)$. Both facts pointed at successful magnetically guided 
targeting of the sample $\mathrm{C}_{6} \mathrm{~S}$ to tumor.

In order to validate this effect of external magnet application and to verify that MNP-doped capsules were indeed delivered to tumor tissue, mice were sacrificed after the last MRI acquisition (48 hours after
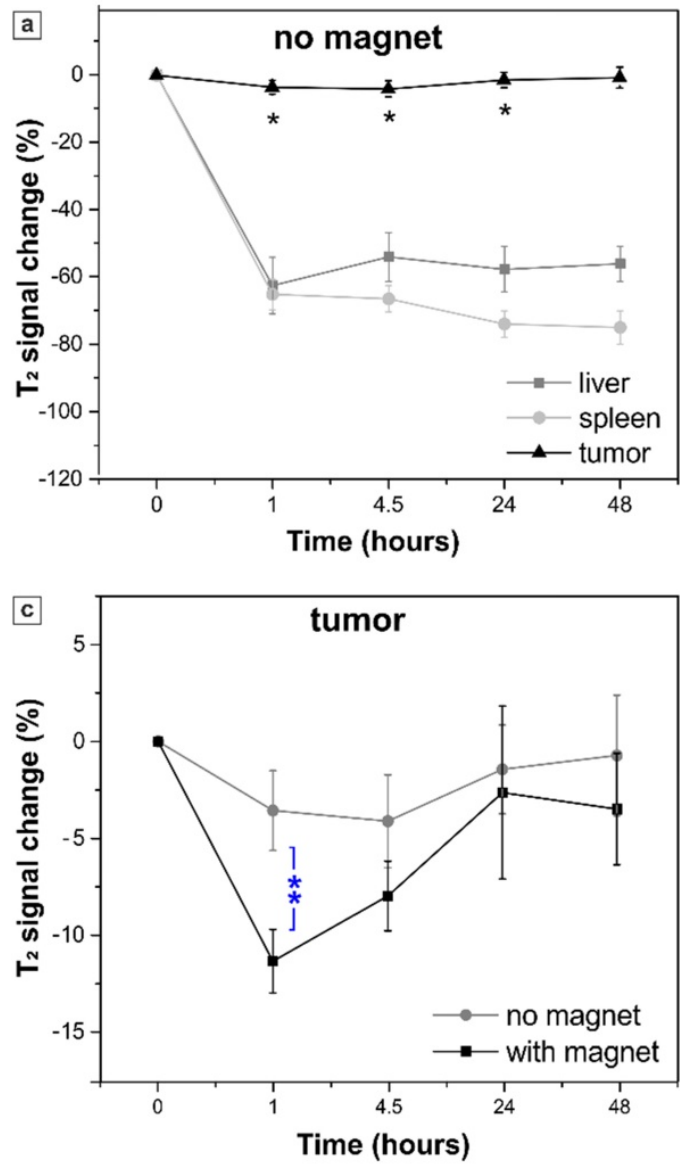

the injection of sample $\mathrm{C}_{6} \mathrm{~S}$ ) to carry out histological analysis of their liver, spleen and tumor. The Perls' (Prussian Blue) staining protocol was used to assess localization of the capsules and iron deposits in tissues. The results are presented in Figure 9.

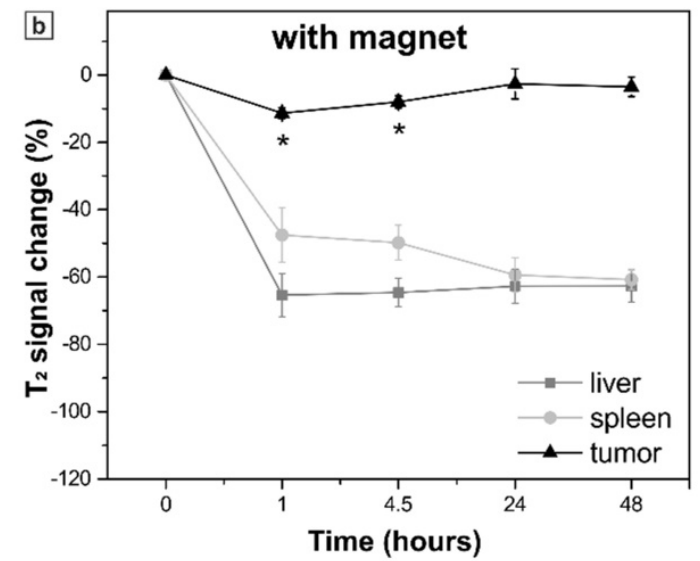

d

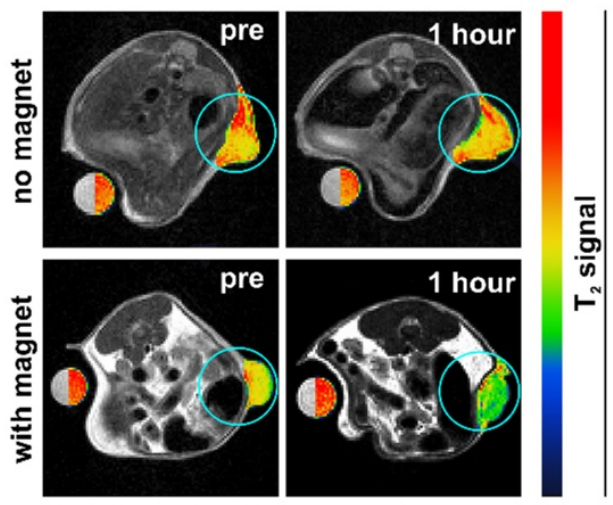
(a) without and (b) with the application of the external magnet to the tumor, an asterisk $\left(^{*}\right)$ indicates significant differences in $T_{2}$ signal change in tumor in comparison to pre-injection images; (c) comparison of $T_{2}$ signal change in the mice tumors with and without magnet application, two asterisks $(* *)$ indicate significant difference $(p<0.01)$ between these two groups. The values correspond to the "mean \pm SE", $n=4$ per each group (no magnet/ with magnet). (d) Representative $T_{2}$-weighted axial MR images of mice acquired before (pre) and 1 hour after the injection. Tumors are marked with blue circles and presented in pseudo colors; a water-containing reference tube is presented in both, original grayscale and pseudo colors.
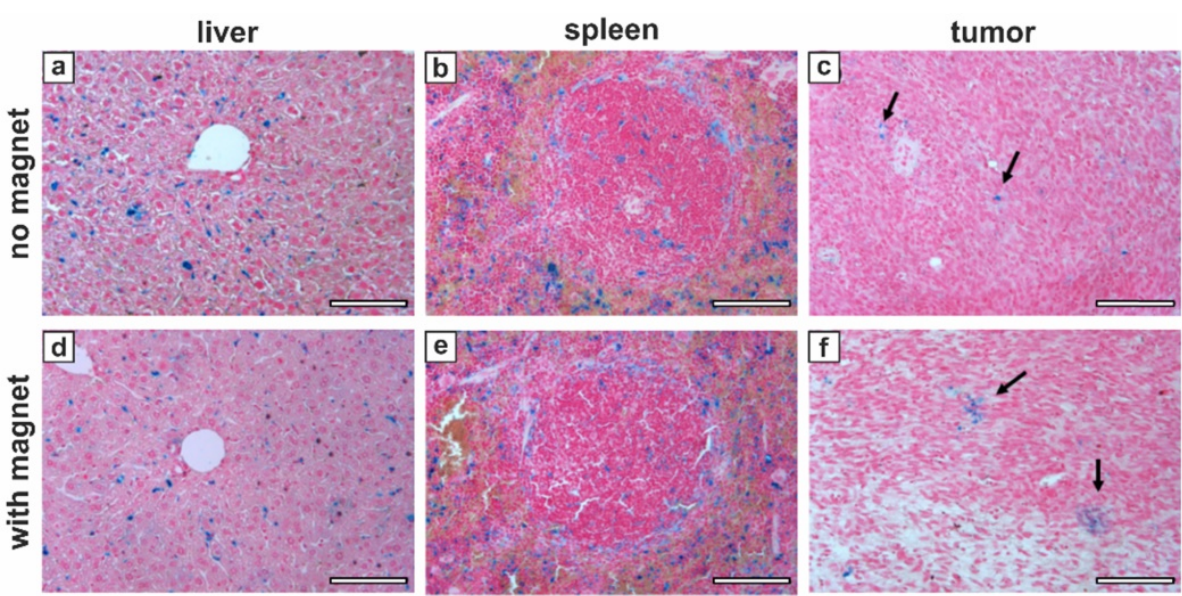

Figure 9. Perls' staining of representative mice tissues 48 hours after the injection of sample $C_{6} S$ without (a-c) and with (d-f) the application of an external magnet: (a, $\left.d\right)-$ liver; (b, e) - spleen; (c, f) - tumor. Black arrows indicate iron deposits in tumor. Scale bars, $100 \mu \mathrm{m}$. 
The Perls' staining positivity of histological sections is shown for all the investigated organs indicating the presence of iron deposits 48 hours after the sample injection (Figure 9), that well matched the MRI results (Figure $8 \mathrm{a}-\mathrm{c}$ ). No marked difference between the groups with and without magnet application was found for liver (Figure 9 a, d) and spleen (Figure $9 \mathrm{~b}, \mathrm{e}$ ) tissues, where the iron was located almost exclusively in macrophages. In tumor, instead, even if the amount of residual contrast agent by this time was limited for both groups, the arrangement of MNP-doped capsules/iron deposits differed significantly (Figure 9 c, f). That clearly shows that an hour-long application of external magnet to tumor following the intravenous injection of the magnetically sensitive capsules resulted in a denser and clustered positioning of the capsules in tumor tissue, as the formed aggregates remained there even 47 hours after removing the magnetic field.

By this means, the obtained results indicated a successful realization of the magnetic targeting using the proposed MNP-doped polyelectrolyte capsules that might guarantee the delivery of a fairly higher amount (at least 3 times, relying on $T_{2}$ contrast observed) of a co-loaded drug into the tumor under external magnetic field. Such enhanced targeted delivery of polyelectrolyte capsules offers great perspectives for drug delivery improvement.

\section{Conclusions}

In summary, the current study highlighted the strategy of magnetic biodegradable polyelectrolyte carriers' design to develop an efficient delivery system combining the externally controlled navigation and MRI visualization properties. The effect of changing the structure of polyelectrolyte/magnetite submicron capsules and the amount of incorporated magnetite has been studied systematically moving from in vitro to in vivo. Incorporation of magnetite nanoparticles into the inner volume of capsules in addition to their shell labeling significantly improved the magnetic targeting ability. Intravenous injection of the most highly loaded sample containing $2.94 \mu \mathrm{mol} F$ (III) to mice with a breast cancer model under external magnet application resulted in a three-fold enhancement in $\mathrm{T}_{2}$ MRI contrast in tumor (compared to the tumor untreated with magnet) together with a pronounced contrast lowering in spleen. Such findings rendered the proposed magnetic capsules effective in terms of both external magnetic field-guided targeting of tumors and MRI monitoring. Summation of these properties of the polyelectrolyte capsules with biocompatibility and the ability of co-loading with an anticancer drug holds the prospect for the effective theranostic platform development aiming at improved anticancer therapy. Further research should be aimed at the application of external physical stimuli (light, ultrasound or alternating magnetic field) providing controlled in-situ drug release or synergetic therapeutic effect in tumor.

\section{Supplementary Material}

Supplementary figures and tables. http://www.ntno.org/v05p0362s1.pdf

\section{Acknowledgements}

The work was supported by the European Commission within Marie Curie Actions "International Research Staff Exchange Scheme" (IRSES-GA-2013-612673). Preparation of magnetic polyelectrolyte capsules, SEM and sedimentation measurements, in vitro cytotoxicity evaluation, as well as in vivo data analysis including quantification of the mean MR signal change, were supported by the Russian Science Foundation (project no 19-73-10123). The authors acknowledge the Italian Ministry of Research for FOE contribution to the Euro-BioImaging MultiModal Molecular Imaging Italian Node (www.mmmi.unito.it). The authors acknowledge the Open Lab of Advanced Microscopy (OLMA) at the Molecular Biotechnology Center (MBC) for support. The authors would like to thank Mr Vsevolod S. Atkin for the SEM measurements, Mr Maxwell A. Astle for the TEM measurements and $\mathrm{Mr}$ Alexey V. Ermakov for the help with graphical illustrations.

\section{Competing Interests}

The authors have declared that no competing interest exists.

\section{References}

1. Mishra DK, Shandilya R, Mishra PK. Lipid based nanocarriers: a translational perspective. Nanomedicine: NBM. 2018; 14: 2023-50.

2. Sreekanth V, Bajaj A. Recent Advances in Engineering of Lipid Drug Conjugates for Cancer Therapy. ACS Biomater Sci Eng. 2019; 5: 4148-66.

3. Molavi F, Barzegar-Jalali M, Hamishehkar H. Polyester based polymeric nano and microparticles for pharmaceutical purposes: A review on formulation approaches. J Control Release. 2020; 320: 265-82.

4. De Cock LJ, De Koker S, De Geest BG, et al. Polymeric Multilayer Capsules in Drug Delivery. Angew Chemie Int Ed. 2010; 49: 6954-73.

5. Cai A, Zhu Y, Qi C. Biodegradable Inorganic Nanostructured Biomaterials for Drug Delivery. Adv Mater Interfaces. 2020; 7: 2000819.

6. Manzano M, Vallet-Regí M. Mesoporous Silica Nanoparticles for Drug Delivery. Adv Funct Mater. 2020; 30: 1902634.

7. Zhou J, Yu G, Huang F. Supramolecular chemotherapy based on host-guest molecular recognition: a novel strategy in the battle against cancer with a bright future. Chem Soc Rev. 2017; 46: 7021-53.

8. Peng L, Liu S, Feng A, Yuan J. Polymeric Nanocarriers Based on Cyclodextrins for Drug Delivery: Host-Guest Interaction as Stimuli Responsive Linker. Mol Pharm. 2017; 14: 2475-86.

9. Busatto S, Walker SA, Grayson W, et al. Lipoprotein-based drug delivery. Adv Drug Deliv Rev. 2020; 159: 377-90. 
10. Thaxton CS, Rink JS, Naha PC, Cormode DP. Lipoproteins and lipoprotein mimetics for imaging and drug delivery. Adv Drug Deliv Rev. 2016; 106: 11631.

11. Sawyer AJ, Kyriakides TR. Matricellular proteins in drug delivery: Therapeutic targets, active agents, and therapeutic localization. Adv Drug Deliv Rev. 2016; 97: 56-68.

12. Farooq MA, Aquib M, Ghayas S, et al. Whey protein: A functional and promising material for drug delivery systems recent developments and future prospects. Polym Adv Technol. 2019; 30: 2183-91.

13. Dissanayake S, Denny WA, Gamage S, Sarojini V. Recent developments in anticancer drug delivery using cell penetrating and tumor targeting peptides. J Control Release. 2017; 250: 62-76.

14. Song Z, Chen X, You X, et al. Self-assembly of peptide amphiphiles for drug delivery: the role of peptide primary and secondary structures. Biomater Sci. 2017; 5: 2369-80.

15. Chung YH, Cai H, Steinmetz NF. Viral nanoparticles for drug delivery, imaging, immunotherapy, and theranostic applications. Adv Drug Deliv Rev. 2020; 156: 214-35.

16. Alemzadeh E, Dehshahri A, Izadpanah K, Ahmadi F. Plant virus nanoparticles: Novel and robust nanocarriers for drug delivery and imaging. Colloids Surfaces B Biointerfaces. 2018; 167: 20-7.

17. Kudela P, Koller VJ, Lubitz W. Bacterial ghosts (BGs) - Advanced antigen and drug delivery system. Vaccine. 2010; 28: 5760-7.

18. Cao Z, Liu J. Bacteria and bacterial derivatives as drug carriers for cancer therapy. J Control Release. 2020; 326: 396-407.

19. Yoo J-W, Irvine DJ, Discher DE, Mitragotri S. Bio-inspired, bioengineered and biomimetic drug delivery carriers. Nat Rev Drug Discov. 2011; 10: 521-35.

20. Villa $\mathrm{CH}$, Anselmo AC, Mitragotri S, Muzykantov V. Red blood cells: Supercarriers for drugs, biologicals, and nanoparticles and inspiration for advanced delivery systems. Adv Drug Deliv Rev. 2016; 106: 88-103.

21. Walker S, Busatto S, Pham A, et al. Extracellular vesicle-based drug delivery systems for cancer treatment. Theranostics. 2019; 9: 8001-17.

22. El-Sawy HS, Al-Abd AM, Ahmed TA, El-Say KM, Torchilin VP. Stimuli-Responsive Nano-Architecture Drug-Delivery Systems to Solid Tumor Micromilieu: Past, Present, and Future Perspectives. ACS Nano. 2018; 12: 10636-64.

23. Sun T, Dasgupta A, Zhao Z, Nurunnabi M, Mitragotri S. Physical triggering strategies for drug delivery. Adv Drug Deliv Rev. 2020; 158: 36-62.

24. Pacardo DB, Ligler FS, Gu Z. Programmable nanomedicine: synergistic and sequential drug delivery systems. Nanoscale. 2015; 7: 3381-91.

25. Torchilin VP. Multifunctional, stimuli-sensitive nanoparticulate systems for drug delivery. Nat Rev Drug Discov. 2014; 13: 813-27.

26. Mi P. Stimuli-responsive nanocarriers for drug delivery, tumor imaging, therapy and theranostics. Theranostics. 2020; 10: 4557-88.

27. Zhu D, Roy S, Liu Z, Weller H, Parak WJ, Feliu N. Remotely controlled opening of delivery vehicles and release of cargo by external triggers. Adv Drug Deliv Rev. 2019; 138: 117-32.

28. Wilhelm S, Tavares AJ, Dai Q, et al. Analysis of nanoparticle delivery to tumours. Nat Rev Mater. 2016; 1: 16014

29. Rosenblum D, Joshi N, Tao W, Karp JM, Peer D. Progress and challenges towards targeted delivery of cancer therapeutics. Nat Commun. 2018; 9: 1410.

30. Blanco E, Shen H, Ferrari M. Principles of nanoparticle design for overcoming biological barriers to drug delivery. Nat Biotechnol. 2015; 33: 941-51.

31. Zhao Z, Ukidve A, Kim J, Mitragotri S. Targeting Strategies for Tissue-Specific Drug Delivery. Cell. 2020; 181: 151-67.

32. Srinivasarao M, Low PS. Ligand-Targeted Drug Delivery. Chem Rev. 2017; 117: 12133-64.

33. Lakshmanan S, Gupta GK, Avci P, et al. Physical energy for drug delivery; poration, concentration and activation. Adv Drug Deliv Rev. 2014; 71: 98-114.

34. Jang D, Jeong J, Song H, Chung SK. Targeted drug delivery technology using untethered microrobots: a review. J Micromechanics Microengineering. 2019; 29: 053002.

35. Polyak B, Friedman G. Magnetic targeting for site-specific drug delivery: applications and clinical potential. Expert Opin Drug Deliv. 2009; 6: 53-70.

36. Liu Y-L, Chen D, Shang P, Yin D-C. A review of magnet systems for targeted drug delivery. J Control Release. 2019; 302: 90-104.

37. Price PM, Mahmoud WE, Al-Ghamdi AA, Bronstein LM. Magnetic Drug Delivery: Where the Field Is Going. Front Chem. 2018; 6: 619.

38. Tietze $\mathrm{R}, \mathrm{Zaloga} \mathrm{J}$, Unterweger $\mathrm{H}$, et al. Magnetic nanoparticle-based drug delivery for cancer therapy. Biochem Biophys Res Commun. 2015; 468: 463-70.

39. Shapiro B, Kulkarni S, Nacev A, Muro S, Stepanov PY, Weinberg IN. Open challenges in magnetic drug targeting. Wiley Interdiscip Rev Nanomedicine Nanobiotechnology. 2015; 7: 446-57.

40. Mody V V., Cox A, Shah S, Singh A, Bevins W, Parihar H. Magnetic nanoparticle drug delivery systems for targeting tumor. Appl Nanosci. 2014; 4: 385-92.

41. Antipina MN, Sukhorukov GB. Remote control over guidance and release properties of composite polyelectrolyte based capsules. Adv Drug Deliv Rev. 2011; 63: 716-29.

42. Voronin D V., Sindeeva OA, Kurochkin MA, et al. In Vitro and in Vivo Visualization and Trapping of Fluorescent Magnetic Microcapsules in a Bloodstream. ACS Appl Mater Interfaces. 2017; 9: 6885-93.

43. Mayorova OA, Sindeeva OA, Lomova M V., et al. Endovascular addressing improves the effectiveness of magnetic targeting of drug carrier. Comparison with the conventional administration method. Nanomedicine: NBM. 2020; 28 . 102184

44. Novoselova M V., German S V., Sindeeva OA, et al. Submicron-Sized Nanocomposite Magnetic-Sensitive Carriers: Controllable Organ Distribution and Biological Effects. Polymers (Basel). 2019; 11: 1082.

45. Anani T, Rahmati S, Sultana N, David AE. MRI-traceable theranostic nanoparticles for targeted cancer treatment. Theranostics. 2021; 11: 579-601.

46. German S V., Bratashov DN, Navolokin NA, et al. In vitro and in vivo MRI visualization of nanocomposite biodegradable microcapsules with tunable contrast. Phys Chem Chem Phys. 2016; 18: 32238-46.

47. Kozlova, German, Atkin, et al. Magnetic Composite Submicron Carriers with Structure-Dependent MRI Contrast. Inorganics. 2020; 8: 11.

48. Golovin YI, Gribanovsky SL, Golovin DY, et al. Towards nanomedicines of the future: Remote magneto-mechanical actuation of nanomedicines by alternating magnetic fields. J Control Release. 2015; 219: 43-60.

49. Kobayashi T. Cancer hyperthermia using magnetic nanoparticles. Biotechnol J. 2011; 6: 1342-7.

50. Hergt R, Dutz S, Müller R, Zeisberger M. Magnetic particle hyperthermia: nanoparticle magnetism and materials development for cancer therapy. J Phys Condens Matter. 2006; 18: S2919-34.

51. Moros M, Idiago-López J, Asín L, et al. Triggering antitumoural drug release and gene expression by magnetic hyperthermia. Adv Drug Deliv Rev. 2019; 138: 326-43.

52. Liu JF, Jang B, Issadore D, Tsourkas A. Use of magnetic fields and nanoparticles to trigger drug release and improve tumor targeting. Wiley Interdiscip Rev Nanomedicine Nanobiotechnology. 2019; 11: e1571.

53. Ruggiero MR, Crich SG, Sieni E, et al. Magnetic hyperthermia efficiency and 1 H-NMR relaxation properties of iron oxide/paclitaxel-loaded PLGA nanoparticles. Nanotechnology. 2016; 27: 285104.

54. Dunne M, Epp-Ducharme B, Sofias AM, Regenold M, Dubins DN, Allen C. Heat-activated drug delivery increases tumor accumulation of synergistic chemotherapies. J Control Release. 2019; 308: 197-208.

55. Donath E, Sukhorukov GB, Caruso F, Davis SA, Möhwald H. Novel Hollow Polymer Shells by Colloid-Templated Assembly of Polyelectrolytes. Angew Chemie Int Ed. 1998; 37: 2201-5.

56. Parakhonskiy B V., Haase A, Antolini R. Sub-Micrometer Vaterite Containers: Synthesis, Substance Loading, and Release. Angew Chemie. 2012; 124: 1221-3.

57. German S V., Novoselova M V., Bratashov DN, et al. High-efficiency freezing-induced loading of inorganic nanoparticles and proteins into micronand submicron-sized porous particles. Sci Rep. 2018; 8: 17763

58. Vaccaro M, Mangiapia G, Paduano L, et al. Structural and Relaxometric Characterization of Peptide Aggregates Containing Gadolinium Complexes as Potential Selective Contrast Agents in MRI. ChemPhysChem. 2007; 8: 2526-38.

59. Nanni P, De Giovanni C, Lollini P-L, Nicoletti G, Prodi G. TS/A: a new metastasizing cell line from a BALB/c spontaneous mammary adenocarcinoma. Clin Exp Metastasis. 1983; 1: 373-80.

60. Xiong R, Soenen SJ, Braeckmans K, Skirtach AG. Towards Theranostic Multicompartment Microcapsules: in-situ Diagnostics and Laser-induced Treatment. Theranostics. 2013; 3: 141-51.

61. Zhao S, Caruso F, Dähne L, et al. The Future of Layer-by-Layer Assembly: A Tribute to ACS Nano Associate Editor Helmuth Möhwald. ACS Nano. 2019; 13: 6151-69.

62. Wuytens P, Parakhonskiy B, Yashchenok A, Winterhalter M, Skirtach A. Pharmacological aspects of release from microcapsules - from polymeric multilayers to lipid membranes. Curr Opin Pharmacol. 2014; 18: 129-40.

63. Volodkin D, Skirtach A, Möhwald H. Bioapplications of light-sensitive polymer films and capsules assembled using the layer-by-layer technique. Polym Int. 2012; 61: 673-9.

64. De Geest BG, Sanders NN, Sukhorukov GB, Demeester J, De Smedt SC. Release mechanisms for polyelectrolyte capsules. Chem Soc Rev. 2007; 36: 636-49

65. Campbell J, Kastania G, Volodkin D. Encapsulation of Low-Molecular-Weight Drugs into Polymer Multilayer Capsules Templated on Vaterite $\mathrm{CaCO} 3$ Crystals. Micromachines. 2020; 11: 717.

66. Ermakov A V., Verkhovskii RA, Babushkina I V, et al. In Vitro Bioeffects of Polyelectrolyte Multilayer Microcapsules Post-Loaded with Water-Soluble Cationic Photosensitizer. Pharmaceutics. 2020; 12: 610.

67. Trushina DB, Akasov RA, Khovankina A V., Borodina TN, Bukreeva T V., Markvicheva EA. Doxorubicin-loaded biodegradable capsules: Temperature induced shrinking and study of cytotoxicity in vitro. J Mol Liq. 2019; 284: 21524.

68. Sharma V, Vijay J, Ganesh MR, Sundaramurthy A. Multilayer capsules encapsulating nimbin and doxorubicin for cancer chemo-photothermal therapy. Int J Pharm. 2020; 582: 119350.

69. Jewell C, Lynn D. Multilayered polyelectrolyte assemblies as platforms for the delivery of DNA and other nucleic acid-based therapeutics. Adv Drug Deliv Rev. 2008; 60: 979-99.

70. Borodina TN, Markvicheva EA, Kunizhev S, Möhwald H, Sukhorukov GB, Kreft O. Controlled Release of DNA from Self-Degrading Microcapsules. Macromol Rapid Commun. 2007; 28: 1894-9.

71. Tarakanchikova Y, Alzubi J, Pennucci V, et al. Biodegradable Nanocarriers Resembling Extracellular Vesicles Deliver Genetic Material with the Highest Efficiency to Various Cell Types. Small. 2020; 16: 1904880

72. Tong W, Gao C. Multilayer Microcapsules with Tailored Structures and Properties as Delivery Carriers for Drugs and Growth Factors. In: Polymeric 
Biomaterials for Tissue Regeneration. Singapore: Springer Singapore;. 2016; p: 75-99.

73. She Z, Wang C, Li J, Sukhorukov GB, Antipina MN. Encapsulation of Basic Fibroblast Growth Factor by Polyelectrolyte Multilayer Microcapsules and Its Controlled Release for Enhancing Cell Proliferation. Biomacromolecules. 2012; 13: 2174-80.

74. De Geest BG, Willart MA, Hammad H, et al. Polymeric Multilayer Capsule-Mediated Vaccination Induces Protective Immunity Against Cancer and Viral Infection. ACS Nano. 2012; 6: 2136-49.

75. De Geest BG, Willart MA, Lambrecht BN, et al. Surface-Engineered Polyelectrolyte Multilayer Capsules: Synthetic Vaccines Mimicking Microbial Structure and Function. Angew Chemie Int Ed. 2012; 51: 3862-6.

76. Chiu Y-C, Gammon JM, Andorko JI, Tostanoski LH, Jewell CM. Assembly and Immunological Processing of Polyelectrolyte Multilayers Composed of Antigens and Adjuvants. ACS Appl Mater Interfaces. 2016; 8: 18722-31.

77. Yanina IY, Svenskaya YI, Prikhozhdenko ES, et al. Optical monitoring of adipose tissue destruction under encapsulated lipase action. J Biophotonics. 2018; 11: e201800058.

78. Lomova M V., Sukhorukov GB, Antipina MN. Antioxidant Coating of Micronsize Droplets for Prevention of Lipid Peroxidation in Oil-in-Water Emulsion. ACS Appl Mater Interfaces. 2010; 2: 3669-76.

79. De Koker S, De Cock LJ, Rivera-Gil P, et al. Polymeric multilayer capsules delivering biotherapeutics. Adv Drug Deliv Rev. 2011; 63: 748-61.

80. Andreeva DV. Polyelectrolyte multilayers for drug delivery. In: Advances and Avenues in the Development of Novel Carriers for Bioactives and Biological Agents. Elsevier. 2020; p: 183-209.

81. Van der Meeren L, Li J, Konrad M, Skirtach AG, Volodkin D, Parakhonskiy B V. Temperature Window for Encapsulation of an Enzyme into Thermally Shrunk, CaCO 3 Templated Polyelectrolyte Multilayer Capsules. Macromol Biosci. 2020; 20: 2000081.

82. Van der Meeren L, Li J, Parakhonskiy B V Krysko D V Skirtach AG. Classification of analytics, sensorics, and bioanalytics with polyelectrolyte multilayer capsules. Anal Bioanal Chem. 2020; 412: 5015-29.

83. Barrefelt $\AA A$, Brismar TB, Egri $G$, et al. Multimodality imaging using SPECT/CT and MRI and ligand functionalized 99mTc-labeled magnetic microbubbles. EJNMMI Res. 2013; 3: 12.

84. Ahmed M, Gustafsson B, Aldi S, et al. Molecular Imaging of a New Multimodal Microbubble for Adhesion Molecule Targeting. Cell Mol Bioeng. 2019; $12: 15-32$.

85. Yi Q, Li D, Lin B, et al. Magnetic Resonance Imaging for Monitoring of Magnetic Polyelectrolyte Capsule In Vivo Delivery. Bionanoscience. 2014; 4: $59-70$.

86. Kozlova AA, German S V, Novoselova M V, et al. Polyelectrolyte Parg/DS submicrocapsules functionalized by magnetite nanoparticles as effective MR contrast agents. J Phys Conf Ser. 2018; 1092: 012067.

87. Alford A, Rich M, Kozlovskaya V, et al. Ultrasound-Triggered Delivery of Anticancer Therapeutics from MRI-Visible Multilayer Microcapsules. Adv Ther. 2018; 1: 1800051.

88. Grillo R, Gallo J, Stroppa DG, et al. Sub-Micrometer Magnetic Nanocomposites: Insights into the Effect of Magnetic Nanoparticles Interactions on the Optimization of SAR and MRI Performance. ACS Appl Mater Interfaces. 2016; 8: 25777-87.

89. Massart R. Preparation of aqueous magnetic liquids in alkaline and acidic media. IEEE Trans Magn. 1981; 17: 1247-8.

90. German S V., Inozemtseva OA, Markin A V Metvalli K, Khomutov GB, Gorin DA. Synthesis of magnetite hydrosols in inert atmosphere. Colloid J. 2013; 75: 483-6.

91. Jain JP, Yenet Ayen W, Domb AJ, Kumar N. Biodegradable Polymers in Drug Delivery. In: Biodegradable Polymers in Clinical Use and Clinical Development. Hoboken, NJ, USA: John Wiley \& Sons, Inc. 2011; p: 1-58.

92. De Koker S, De Geest BG, Cuvelier C, et al. In vivo Cellular Uptake, Degradation, and Biocompatibility of Polyelectrolyte Microcapsules. Adv Funct Mater. 2007; 17: 3754-63.

93. De Geest BG, Vandenbroucke RE, Guenther AM, et al. Intracellularly Degradable Polyelectrolyte Microcapsules. Adv Mater. 2006; 18: 1005-9.

94. Valdepérez D, del Pino P, Sánchez L, Parak WJ, Pelaz B. Highly active antibody-modified magnetic polyelectrolyte capsules. J Colloid Interface Sci. 2016; 474: 1-8.

95. Vidiasheva I V , Abalymov AA, Kurochkin MA, et al. Transfer of cells with uptaken nanocomposite, magnetite-nanoparticle functionalized capsules with electromagnetic tweezers. Biomater Sci. 2018; 6: 2219-29.

96. Laurent S, Forge D, Port $M$, et al. Magnetic Iron Oxide Nanoparticles: Synthesis, Stabilization, Vectorization, Physicochemical Characterizations, and Biological Applications. Chem Rev. 2008; 108: 2064-110.

97. Abbasi AZ, Gutiérrez L, del Mercato LL, et al. Magnetic Capsules for NMR Imaging: Effect of Magnetic Nanoparticles Spatial Distribution and Aggregation. J Phys Chem C. 2011; 115: 6257-64.

98. Feng Q, Liu Y, Huang J, Chen K, Huang J, Xiao K. Uptake, distribution, clearance, and toxicity of iron oxide nanoparticles with different sizes and coatings. Sci Rep. 2018; 8: 2082

99. ISO 10993-5 Biological evaluation of medical devices - Part 5: Tests for in vitro cytotoxicity in vitro cytotoxicity. International Organization for Standardization Geneva. 2009

100. Torchilin V. Tumor delivery of macromolecular drugs based on the EPR effect. Adv Drug Deliv Rev. 2011; 63: 131-5.
101. Shojaee P, Niroomand-Oscuii $\mathrm{H}$, Sefidgar M, Alinezhad L. Effect of nanoparticle size, magnetic intensity, and tumor distance on the distribution of the magnetic nanoparticles in a heterogeneous tumor microenvironment. J Magn Magn Mater. 2020; 498: 166089.

102. Baroni S, Ruggiero MR, Bitonto V, et al. In vivo assessment of tumour associated macrophages in murine melanoma obtained by low-field relaxometry in the presence of iron oxide particles. Biomaterials. 2020; 236: 119805 . 